\title{
Imaging brain microstructure with diffusion MRI: Practicality and applications
} practicality and applications

\author{
Alexander, Daniel C.; Dyrby, Tim Bjørn; Nilsson, Markus; Zhang, Hui
}

Published in:

N M R in Biomedicine

Link to article, DOI:

10.1002/nbm.3841

Publication date:

2018

Document Version

Peer reviewed version

Link back to DTU Orbit

Citation (APA):

Alexander, D. C., Dyrby, T. B., Nilsson, M., \& Zhang, H. (2018). Imaging brain microstructure with diffusion MRI: Practicality and applications: practicality and applications. N M R in Biomedicine, 32(4), [e3841].

https://doi.org/10.1002/nbm.3841

\section{General rights}

Copyright and moral rights for the publications made accessible in the public portal are retained by the authors and/or other copyright owners and it is a condition of accessing publications that users recognise and abide by the legal requirements associated with these rights.

- Users may download and print one copy of any publication from the public portal for the purpose of private study or research.

- You may not further distribute the material or use it for any profit-making activity or commercial gain

- You may freely distribute the URL identifying the publication in the public portal 


\title{
Imaging brain microstructure with diffusion MRI: practicality and applications
}

\author{
Daniel C. Alexander ${ }^{1}$ (D) । Tim B. Dyrby ${ }^{2,3}$ (i) | Markus Nilsson ${ }^{4}$ (i) | Hui Zhang ${ }^{1}$
}

${ }^{1}$ Centre for Medical Image Computing (CMIC), Department of Computer Science, UCL (University College London), Gower Street, London, UK

${ }^{2}$ Danish Research Centre for Magnetic Resonance, Center for Functional and Diagnostic Imaging and Research, Copenhagen University Hospital Hvidovre, Hvidovre, Denmark

${ }^{3}$ Department of Applied Mathematics and Computer Science, Technical University of Denmark, Kongens Lyngby, Denmark

${ }^{4}$ Clinical Sciences Lund, Department of Radiology, Lund University, Lund, Sweden

Correspondence

Daniel C. Alexander, Centre for Medical Image Computing (CMIC), Department of Computer Science, UCL (University College London), Gower Street, London WC1E 6BT, UK. Email: d.alexander@ucl.ac.uk

Funding information

Engineering and Physical Sciences Research Council, Grant/Award Number: EP/L022680/ 1, EP/M020533/1 and EP/N018702/1; Scleroseforeningen, Grant/Award Number: A31910; Region Hovedstaden, Grant/Award Number: A5657; Vetenskapsrådet, Grant/ Award Number: 2016-03443; Stiftelsen för Strategisk Forskning, Grant/Award Number: AM13-0090

\section{1 | INTRODUCTION}

The central vision in microstructure imaging is of virtual histology: estimating and mapping histological features of tissue using non-invasive imaging techniques, such as MRI. This virtual histology has several advantages over classical histology: (i) it is non-invasive, avoiding the need for tissue samples, e.g. from biopsy; (ii) it views intact in situ tissue, avoiding disruptions that arise from tissue extraction and preparation; (iii) it is non-destructive, so enables repeat measurements for monitoring; (iv) it provides a wide field of view, typically showing a whole organ or body, rather than the small samples often used in classical histology; and ( $v$ ) data acquisition is relatively fast, cheap and automated compared with classical histology.

Abbreviations: ADD, axon diameter distribution; AQP-4, Aquaporin-4; AIC, Akaike's information criterion; BIC, Bayesian information criterion; CC, corpus callosum; CHARMED, composite hindered and restricted models of diffusion; CODIVIDE, COnstrained DIffusional Varlance DEcomposition; DIC, differential interference contrast; DDE, double diffusion encoding; DIVIDE, DIffusional Varlance DEcomposition; DODE, double oscillating diffusion encoding; DTI, diffusion tensor imaging; DKI, diffusion kurtosis imaging; DSI, diffusion spectrum imaging; DBSI, diffusion basis spectrum imaging; ECS, extracellular, space; FEXI, filter exchange imaging; GM, grey matter; HARDI, high-angular-resolution diffusion imaging; IVIM, intra voxel incoherent motion; MAP, mean apparent propagator; MMWMD, minimal model of white matter diffusion; MD, mean diffusivity; MCMC, Markov chain Monte Carlo; MC, Monte Carlo; NODDI, neurite orientation dispersion and density imaging; NAWM, normal appearing white matter; OD, orientation dispersion; ODE, Oscillating diffusion encoding; OGSE, oscillating gradient spin echo; PGSE, pulsed gradient spin echo; PGSTE, pulsed-gradient stimulated-echo sequence; QTE, $q$-trajectory encoding; SDE, single diffusion encoding; SE, spin echo; STE, stimulated echo; TE, echo time; TR, repetition time; TDE, triple diffusion encoding; vic, neurite density index; viso, isotropic fraction; WM, white matter All authors contributed equally and are listed alphabetically. 
Classical histology has been a lynchpin in the development of modern neuroscience including understanding the brain's macroscopic organization (see, e.g., Reference 1), the mechanisms of connectivity and communication, ${ }^{2}$ and the pathologies underpinning neurodegeneration. ${ }^{3}$ Such work primarily uses sliced post-mortem tissue. Clinical applications in the brain are mostly for post-mortem confirmation of diagnosis, as in vivo brain biopsy is normally justified only in aggressive diseases such as grading brain tumours. The non-invasive, non-destructive nature of virtual histology offers the potential to study the live brain in situ in healthy volunteers or patients. The relative ease of data acquisition allows population studies that provide insight into anatomical variability. Furthermore, its non-destructive nature allows repeat measurements to monitor changes during normal development or pathological processes. Clinically, virtual histology avoids biopsy and the potential side effects of the invasive procedure, and provides a window on tissue changes when the risk of side effects prohibits biopsy. Moreover, the wide field of view that virtual histology provides potentially reduces false negatives that may arise from, say, poor targeting of a biopsy.

Figure 1 compares typical images from classical histology and microstructure imaging. The clear advantage of classical histology is its level of anatomical detail; its submicrometre image resolution provides vivid insight into the cellular architecture of tissue, whereas microstructure imaging
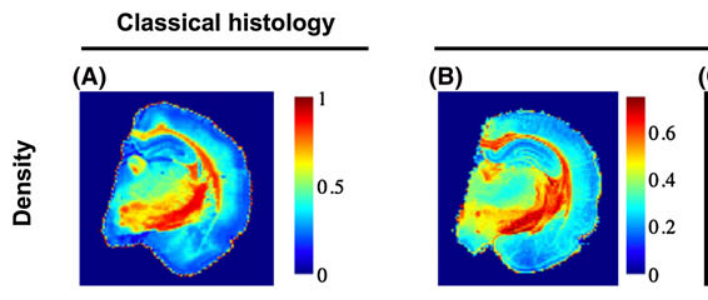

(F)

(E)

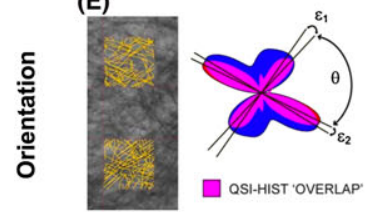

(H)

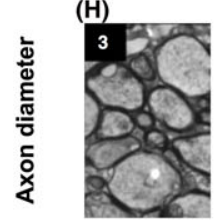

(M)

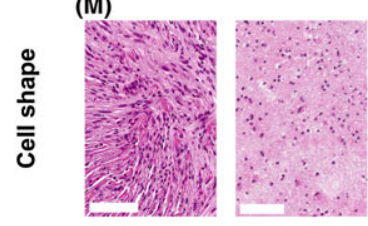

(Q)

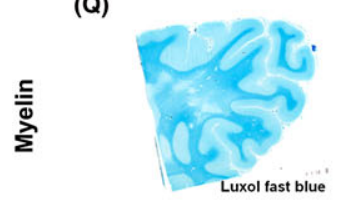

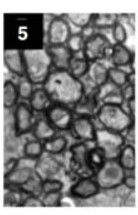

(I)

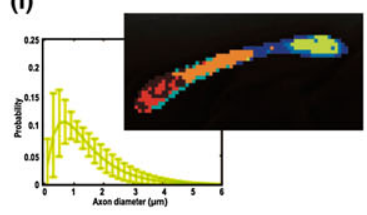

(N)

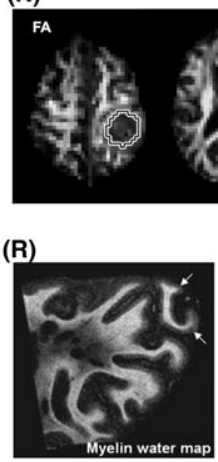

(S)
Microstructure imaging by MRI
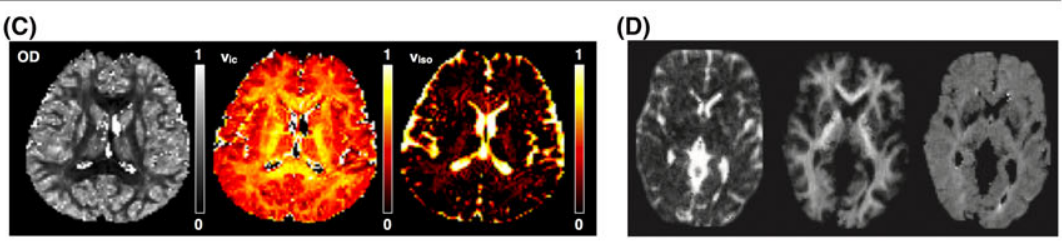

(G)
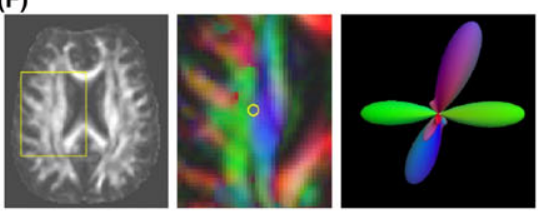

(J)

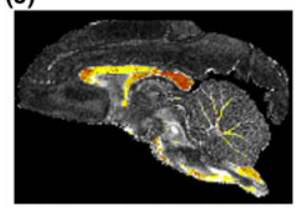

(K)

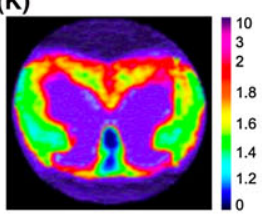

(L)

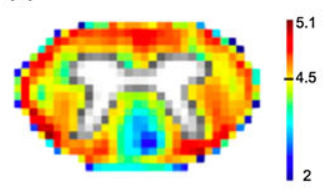

(P)
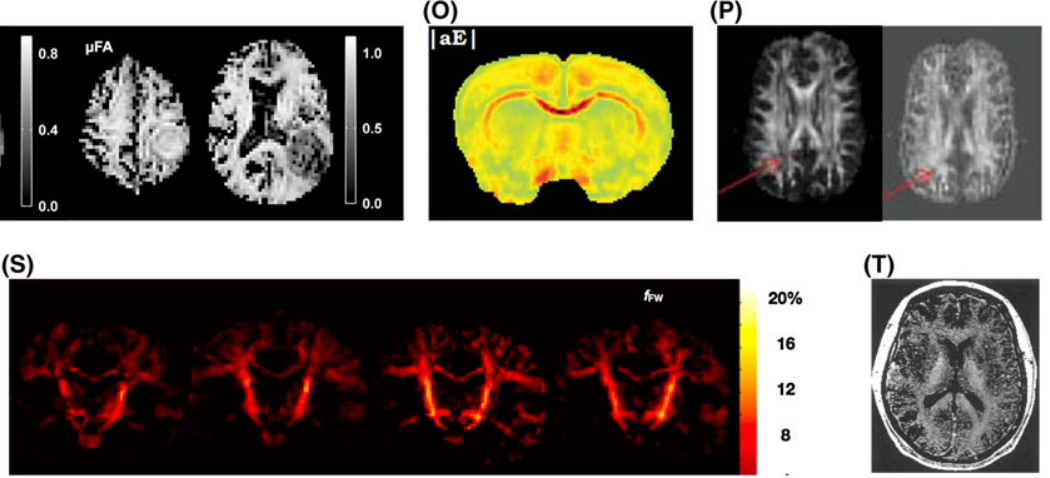

FIGURE 1 Comparison of classical histology and microstructure imaging showing a range of microstructure imaging techniques in the current literature organized by target tissue feature. A-D, Imaging indices of neurite (axon or dendrite) density with classical histology from Reference 4 $(A)$ and by model-based dMRI (B-D). Maps show the cylinder fraction from Reference 4 (B), orientation dispersion (OD), neurite density index ( $\left.v_{i c}\right)$ and isotropic fraction $\left(v_{\text {iso }}\right)$ from NODDI $(C),{ }^{5}$ and isotropic fraction, 'stick density', and tissue mean diffusion from CODIVIDE (D). ${ }^{6}$ E-G, Imaging fibre orientation distribution. E, Estimation of fibre directions from histology and corresponding estimates from dMRI. ${ }^{7} \mathrm{~F}$, In vivo fibre orientation mapping using constrained spherical convolution. ${ }^{8} \mathrm{G}$, Combined mapping of microstructure and orientation by the spherical mean technique. ${ }^{9} \mathrm{H}$-L, Imaging indices of axon diameter. $\mathrm{H}$, Histology provides high-resolution maps enabling measurements of individual axon diameters; images from Reference 10. I, Estimated axon diameter distributions from diffusion MRI using AxCaliber in Reference 10 of the in vivo rat-brain cluster into groups reflecting corresponding diameter histograms from histology. J-L, Axon diameter indices from the monkey brain using ActiveAx (J), ${ }^{11}$ ex vivo spinal cord $(\mathrm{K})^{12}$ and in vivo spinal cord using $300 \mathrm{mT} / \mathrm{m}$ gradients $(\mathrm{L}){ }^{13} \mathrm{M}-\mathrm{P}$, Imaging cell shape indices. $\mathrm{M}$, Classical histology reveals elongated cells in a meningioma to the left and rounder cells in a glioma to the right; from Reference 14 . N, Fractional anisotropy from DTI is low in both meningioma and glioma tumours, but the microscopic anisotropy ( $\mu$ FA) from DIVIDE is more specific to cell shape and shows high value in the meningioma only. ${ }^{14} \mathrm{O}, \mathrm{P}, \mathrm{A}$ similar measure of the microscopic anisotropy from double diffusion encoding in a rat brain (O) ${ }^{15}$ and a healthy human brain (P). ${ }^{16} \mathrm{Q}-\mathrm{T}$, Imaging myelin density. Classical histology by luxol fast blue shows reduced myelin density in the brain of a multiple sclerosis patient (Q) and MRI-derived maps using quantitative relaxometry show similar features (R). ${ }^{17} \mathrm{~S}, \mathrm{MRI}$ used to track the myelination in infants. ${ }^{18} \mathrm{~T}$, An early example of the myelin water fraction from relaxation-weighted MRI ${ }^{19}$ 


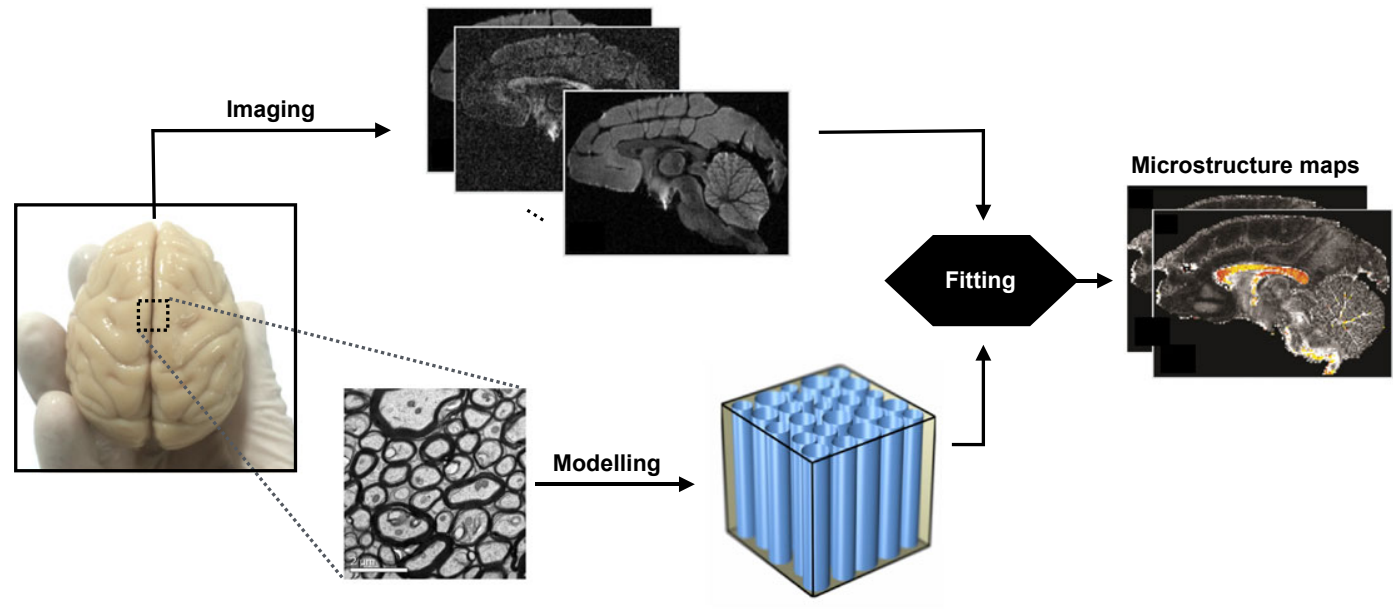

FIGURE 2 Illustration of the microstructure imaging paradigm, which fits a model relating microscopic tissue features to MR signals in each voxel to produce microstructure maps. For example, various techniques to map indices of axon diameter ${ }^{10-12,20,21}$ use a simple geometric model of white matter microstructure, consisting of parallel non-abutting impermeable cylinders that represent axons. The methods acquire a set of images with varying diffusion weighting and fit the model in each voxel to recover estimates of cylinder size and packing density, which provide maps of indices of axon diameter and axon density. MRI maps from Reference 11. Electron microscopy courtesy of Mark Burke

provides only statistical descriptions of the tissue over the extent of millimetre-sized image voxels. In some applications, rich and specific content of classical histological images is important: for example, in enabling a cancer histopathologist to identify the presence of minute fractions of mitotic cells. However, many tasks that histologists perform seek broader statistical changes over a relatively wide extent of tissue. For example, the density and diameter distribution of axons in a white matter pathway determine its information-bearing capacity; different densities, shapes and configurations of cells discriminate different types of brain tumour; widespread protein deposits are hallmarks of Alzheimer's disease. In such applications, the precise detail of cellular architecture is less important and the benefits of microstructure imaging can significantly outweigh those of traditional histology.

Microstructure imaging relies on a model that relates microscopic features of tissue architecture to MR signals. In general, the approach acquires a set of images with different sensitivities and fits a model in each voxel to the set of signals obtained from the corresponding voxel in each image. The process yields a set of model parameters in each image voxel, which constitute parameter maps of microscopic tissue features. Figure 2 illustrates with an example based on diffusion MRI.

Diffusion MRI is a key modality for microstructure imaging, because of its unique sensitivity to cellular architecture. The technique sensitizes the MR signal to the random dispersion of signal-bearing particles, typically water molecules, over diffusion times from the millisecond range up to around one second. The mean free path over this time at room or body temperature is in the micrometre range, i.e. the cellular scale, so the cellular architecture of the tissue strongly influences the dispersion pattern of the molecules. Thus diffusion MR measurements support inferences on tissue microstructure.

This article reviews the current state of the art in microstructure imaging of the brain using diffusion MRI. We thus focus on diffusion MRI techniques that aim to estimate and map tissue properties via biophysical models and mention only in passing diffusion MRI techniques based on signal models, which other parts of this special issue cover in more detail. ${ }^{22}$ This kind of technique has reached an important turning point in recent years with its transformation from largely a technical research topic to widespread application in biomedical studies. With this in mind, the review aims to emphasize practicalities of developing microstructure-imaging techniques designed for front-line application while giving a critical review of the state of the art. Thus Section 2 provides some background information on brain anatomy at the scale we are sensitive to with diffusion MRI together with the nature of the measurements we make. Section 3 then reviews the state of the art in models underpinning current microstructure imaging techniques and the range of current applications. Section 4 focuses on practical issues in the development of microstructure imaging techniques, specifically model selection, experiment design, parameter estimation and validation. That section concludes with an outline of the microstructure-imaging development pipeline. Finally, Section 5 discusses the future of diffusion MRI microstructure imaging of the brain highlighting opportunities for future research, development and application, and considers the wider perspective of applications outside the brain and exploiting contrasts other than diffusion MRI.

\section{2 | BACKGROUND}

This section provides some background context for the rest of the article. First it provides information on the anatomy of brain tissue at the cellular scale-the primary targets for diffusion MRI and microstructure imaging. Second it reviews the range of diffusion MR measurements available to probe this anatomy. 

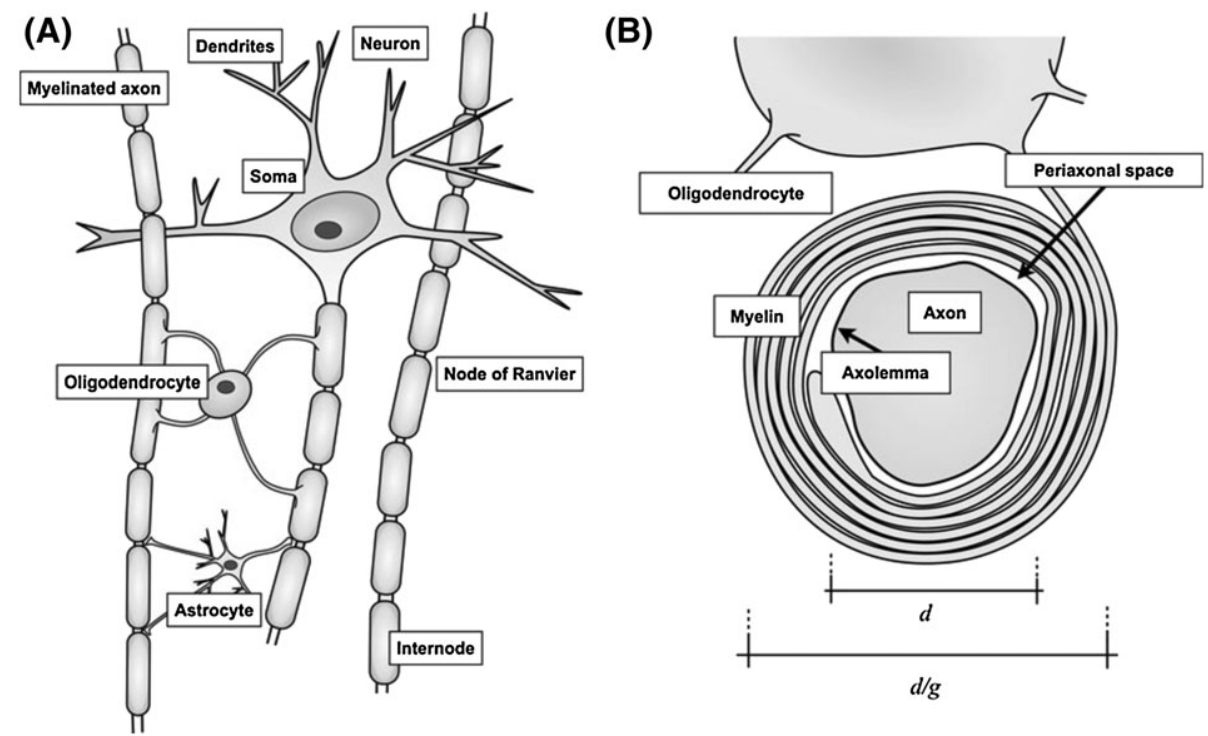

FIGURE 3 The neuron and the glial cells in brain tissue. A, Cells have a cell body or 'soma'. All cells have processes that extend from their soma, but only the neuron has an axon. Glial cells-astrocytes, oligodendrocytes, and microglia-support neuronal function. Oliogodendrocytes associate with several axons to wrap around each a segment, called an 'internode', of the thin layer of fatty myelin to boost saltatory conduction. The nodes of Ranvier are the gaps between internodes. B, The thickness of the myelin around axons in normal conditions has a roughly constant ratio with the axon diameter. This ratio is called the g-ratio (defined in fact as the inner diameter divided by the outer diameter). The space encapsulated by the axolemma, the cell membrane surrounding the axon, is the intra-axonal space. The illustration is reproduced from Reference 23

\section{1 | Brain microanatomy}

Brain tissue contains neurons and glial cells and is separated into two types: grey matter (GM) and the white matter (WM). The GM contains the cell bodies, i.e. somas of neurons and glial cells, as well as neuronal dendrites, short-range intra-cortical axons and the stems of long-range axons extending into the white matter. The WM is dominated by densely packed and often myelinated axons that emanate from the soma of neurons in GM, and project to distal GM areas or other parts of the body. Glial cells are also found in WM. Figure 3 illustrates the neuron and its environment, which in combination provide the basic mechanisms for brain function via communication between brain regions. ${ }^{24}$

\subsection{1 | Dendrites}

In grey matter, the dendrites range from 0.2 to $3 \mu \mathrm{m}$ in diameter for dendrites both proximal and distal to the soma of the neuron. ${ }^{25}$ The dendrites branch from the soma membrane in a formation like a tree crown; see Figure 4.

The structure of the dendritic tree and its branches, their extent and architectural outline depends on the type of neuron. ${ }^{25}$ In general, relatively few (typically one to five) dendrites extend from the soma body itself, but the total number of branches varies from four to more than 400. The cerebellar Purkinje cells have the most branches. The spatial extent of the dendrite tree ranges from 15 to $1800 \mu \mathrm{m}$ radial distance from the soma to the tip of the most distal dendrite. Dendrites from each neuron strongly intermingle with those from other neurons in their neighbourhood to form a dense and complex dendritic network. ${ }^{25}$ The Golgi-Cox stain, as shown in Figure 4, visualizes just a fraction of neurons so does not reveal the full complexity of the dendritic network, but does highlight the variety of shapes of the dendritic tree. Figure $4 \mathrm{~F}$ also shows small protrusions from the main shaft of the dendrites, which are called dendritic spines; see Reference 30 for a review of their structure and function. For neurons in the cerebral cortex, the dendritic trees are mostly isotropic, extending and branching evenly in all directions, whereas elsewhere, e.g. in the layers of the hippocampus, the trees can be highly anisotropic to support inter-layer connectivity. ${ }^{25,26}$

\subsection{2 | Axons}

The connecting distances of axons range from a few millimetres, e.g. for intra-cortical connections, to over 1 m, e.g. for the corticospinal connections in humans. ${ }^{31}$ In WM the axon diameter ranges from $0.1 \mu \mathrm{m}$ to more than $10 \mu \mathrm{m}$ in vertebrates, with myelinated axon diameters rarely less than about $0.2 \mu \mathrm{m}$. A small number of 'giant' axons ( $>3 \mu \mathrm{m})$ arise in the vertebrate brain, and they have been observed for example in the corpus callosum $(\mathrm{CC})^{32,33}$ and cortico-spinal tracts. ${ }^{34}$ Larger axons transmit signals more quickly, as saltatory conduction, i.e. the signal propagation along the axon, increases in speed approximately linearly with axon diameter. ${ }^{35}$ However, space constraints within the brain make giant axons 'expensive' to host. ${ }^{36}$ Moreover, energy consumption increases super-linearly with axon diameter, further favouring small axons. ${ }^{36}$

A brain connection is formed by a bundle of axons in WM that share a trajectory from emanation to target region. The axon diameter distribution (ADD) of a brain connection largely depends on the target region, ${ }^{37,38}$ and varies among species for the same connection. ${ }^{39}$ However, the ADD often has shape similar to a gamma distribution, as often assumed in mathematical models, e.g. References 20 and 40 , i.e. a single-mode single-sided (positive only) distribution with a heavy tail extending into the range of 'giant' axons. The mean of an ADD that contains myelinated axons 


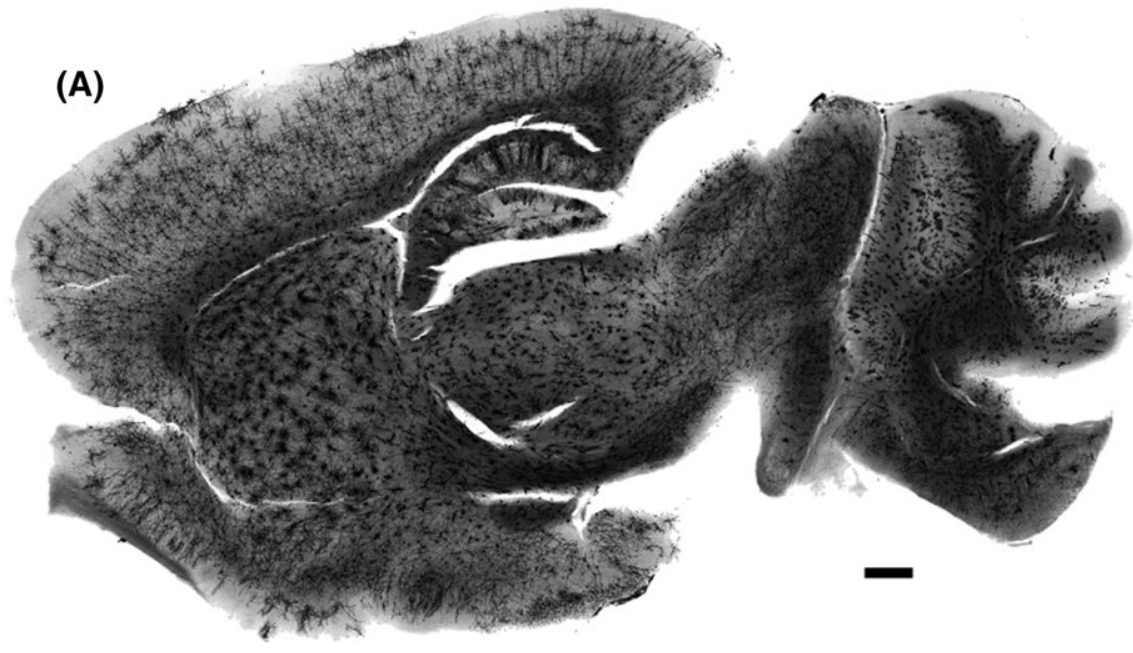

FIGURE 4 Golgi-Cox staining of the adult mouse brain to highlight neuronal and dendritic structures in grey matter. A, Neurons in all brain regions are evenly and reliably stained with a Golgi-Cox protocol. B-E, Magnified images of cerebral cortex (B,C), hippocampus (D), and cerebellar cortex (E). F, Dendritic spines are visible at high magnification. (Golgi staining, differential interference contrast (DIC) images, scale bars $500 \mu \mathrm{m}$ in $\mathrm{A}, \mathrm{B}, 50 \mu \mathrm{m}$ in $\mathrm{E}$ and $5 \mu \mathrm{m}$ in $\mathrm{F}$.) Figure and modified text from Reference 26, Figure 5
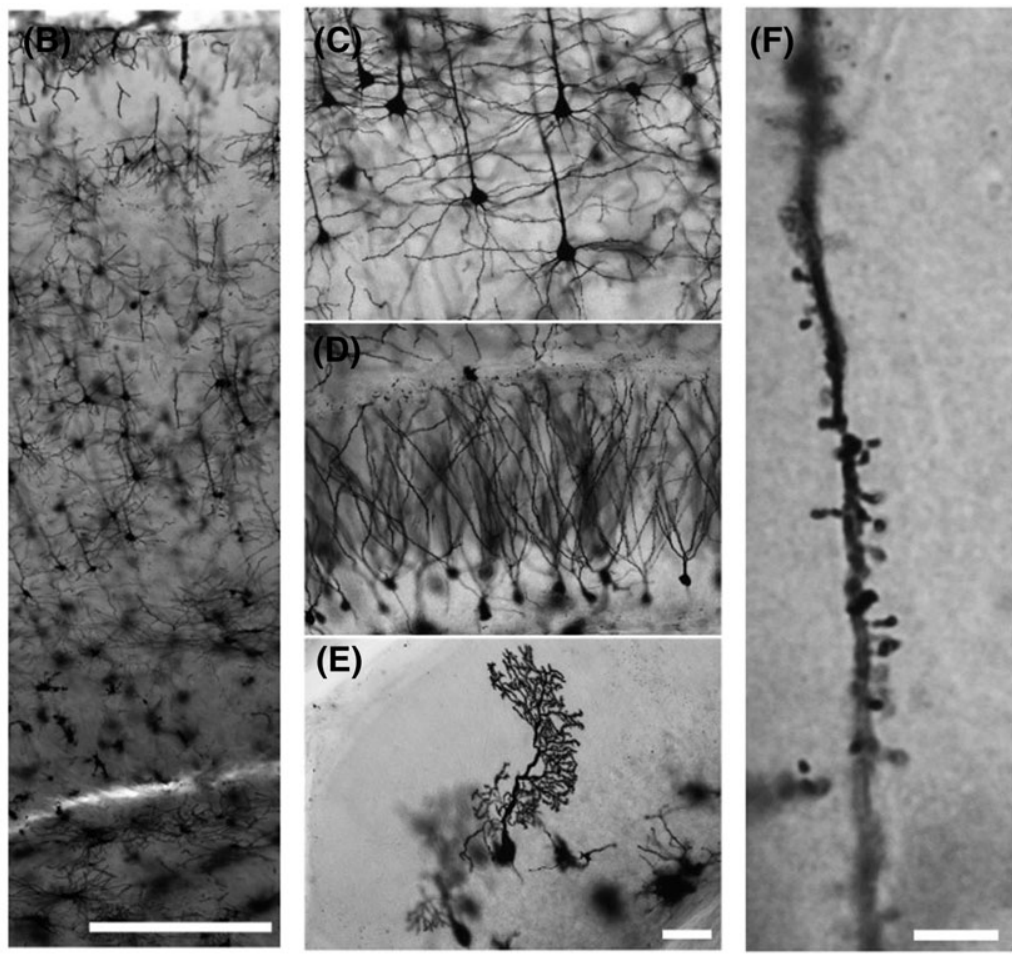

is typically 0.5-0.8 $\mu \mathrm{m}$. Most ADD measurements are reported in the mid-sagittal CC. There, the mid-body of the CC has a larger mean ADD than the genu, and the splenium has the smallest mean ADD (even though occasional giant axons do appear). ${ }^{32,33,39,41}$ In mammals, brain connections that project through the mid-body tend to include the largest diameter axons. ${ }^{39}$ These connections are associated with the motor system, which is one of the fastest conducting systems in the central nervous system and also includes the longest axons in the brain. Since the need for speed of a brain connection, and thus its ADD, is determined by its target, ${ }^{37,38}$ projections with different targets but emanating from the same cortical region $^{42}$ can contain quite different ADDs. For example the corticostriatal projections (ipsi- as well as contralaterally) typically contain smaller axons compared with the corresponding contralateral corticocortical projections. ${ }^{37,43}$ Interestingly, the size of the neuronal soma varies by neuron type in the range 7-58 $\mu \mathrm{m}$, and for some neurons a positive correlation with axon diameter has been found. ${ }^{44,45}$

\subsection{3 | Myelin}

The myelin sheath consists of $80 \%$ lipids and $20 \%$ proteins and wraps around the axon in layers about 10 nm thick, as illustrated in Figure 3 . The myelin sheath divides into segments along the axon with regularly spaced gaps called 'nodes of Ranvier' or just 'nodes'. The internodal distance is approximately proportional to the outer axon diameter (i.e. myelin and axon) with a coefficient of proportionality of about 100 . Thus the segments are 0.2-2 mm long, ${ }^{46}$ whereas the nodes of Ranvier themselves are 1-2 $\mu \mathrm{m}$ long. ${ }^{47}$

The myelin insulates the axon, which boosts the conduction speed along axons by a factor of about 5.5. ${ }^{35}$ The outer diameter of a myelinated axon has an optimal ratio to the inner axon diameter (i.e. without the myelin). The ratio (inner diameter divided by outer diameter) is known as the $g$ ratio, and in normal CNS simulations suggest the g-ratio that optimizes conduction speed is about 0.7 .48 
In the primate CC, the fraction of unmyelinated axons as observed with EM is small compared with myelinated axons. Across the mid-sagittal CC, the largest fraction of unmyelinated axons can be found in the genu (16-20\%), which includes the prefrontal corticocortical projections. ${ }^{32,33}$ The function of unmyelinated axons is still not clearly understood, but Reference 38 provides some thoughts.

\subsection{4 | Intra-axonal structure}

The intra-axonal space shown in Figure 3B is the space encapsulated by the cell membrane of axons and contains macromolecules and proteins, as well as solid filaments and mitochondria. In axons, the cytoskeleton consists of filaments that maintain the axon's shape and internal organization, and acts as mechanical support for the intra-axonal transportation system i.e. the microtubules. The microtubules have a diameter of about $25 \mathrm{~nm}$ and are the intra-axonal railway transporting substances to and from the cell body, both retrograde and anterograde; they are easily seen with EM. The density of microtubules relates to axon diameter but not length. ${ }^{49}$

\subsection{5 | Glial cells}

Besides neurons, the central nervous system also contains different types of glial cell. In contrast to neurons, glial cells have no axon and retain the ability to undergo cell division in adulthood. In adult human neocortex, References 50 and 51 find the proportion of glial cells (by cell count) to be 76.6\% oligodendrocytes, $17.3 \%$ astrocytes and 6.5\% microglia. Moreover, in adults, the glia/neuron ratio is 1.32 and 1.40 for males and females respectively. Aquaporin-4 (AQP-4) water channels in the cell membrane, which make the membrane highly permeable to water, are a feature of glial cells. $^{52}$

Oligodendrocytes create the myelin sheaths around axons, as shown in Figure 3; see Reference 53 . The soma is about $13 \mu \mathrm{m}$ in diameter and extends up to 30 processes each like an arm that embraces a different axon, providing the myelin for one internode segment. The processes extend to distances of $80-120 \mu$. $^{54}$

Astrocytes have somas of about $10 \mu \mathrm{m}$ in diameter from which many processes extend in a star-shaped formation, with an overall diameter of about $150 \mu \mathrm{m} .{ }^{55}$ They perform a plethora of functions, e.g. a role in tissue repair and scarring, as well as maintenance of extracellular ion balance probably in relation to sleep. ${ }^{56}$ The list is constantly growing; see Reference 57 for a review of current understanding. The astrocytes are territorial, meaning that their processes intermingle very little with the processes of neighbouring astrocytes.

Microglia are macrophages that provide the first reaction for many CNS injuries. ${ }^{58}$ Their soma is $10 \mu \mathrm{m}$ in diameter, and total coverage (with processes) is about $15-30 \mu \mathrm{m}$. Like astrocytes, microglia are territorial cells. ${ }^{59}$

\subsection{6 | Interstitial space}

The interstitial, or extracellular, space (ECS) is the space that surrounds anatomical structures such as cells, axons and dendrites. Invasive microscopy techniques suggest that the fraction of ECS in adult brain of various non-human species is $15-35 \% .^{60}$ However, neither electron nor light microscopy can provide reliable measurements of ECS fraction, because chemicals used in the processing of the tissue for the display introduce dehydration. The resulting shrinkage effects have been reported to be as low as less than $1 \%$ and as high as $65 \%{ }^{32,33,41,61}$

\subsection{7 | Macroscopic configurations}

The image resolution of MRI typically provides voxels of a few $\mathrm{mm}^{3}$ in volume, from which we aim to draw microanatomical statistics such as anisotropy, cell sizes and axon diameters-features in the micrometre length scale. Axons often extend across many voxels, and each voxel can contain hundreds of thousands of axons, which can adopt a wide variety of configurations, e.g. bending, fanning, crossing etc., $7,62,63$ as illustrated in Figure 5A. Moreover, axons are not straight even within a single voxel, as Figure 5B illustrates, which further complicates the task of modelling the geometry of axons at the millimetre scale.

\subsection{8 | Vascular system}

The brain contains three vessel systems for blood perfusion: arteries, veins and capillaries. The capillaries contain the smallest vessels and ensure perfusion in brain tissue. They range in diameter between about 5 and $10 \mu \mathrm{m}$, and capillary density in cortical layers is high compared with WM. ${ }^{64}$ Macroscopically, the capillaries generally appear randomly organized, ${ }^{64}$ and when perfused produce a water-dispersion effect, called intra voxel incoherent motion (IVIM), ${ }^{65}$ similar to water diffusion through Brownian motion, although dispersion is more rapid and has a different time dependence.

\section{2 | Sequences}

Diffusion is encoded into the MR signal by time-varying magnetic field gradients. Here, we focus on the most common type of pulse sequence for dMRI, ${ }^{66}$ which yields so-called single diffusion encoding (SDE). ${ }^{67}$ We will also briefly cover other encodings that can overcome some of the limitations inherent to SDE; Figure 6 illustrates the various pulse sequences we consider. 


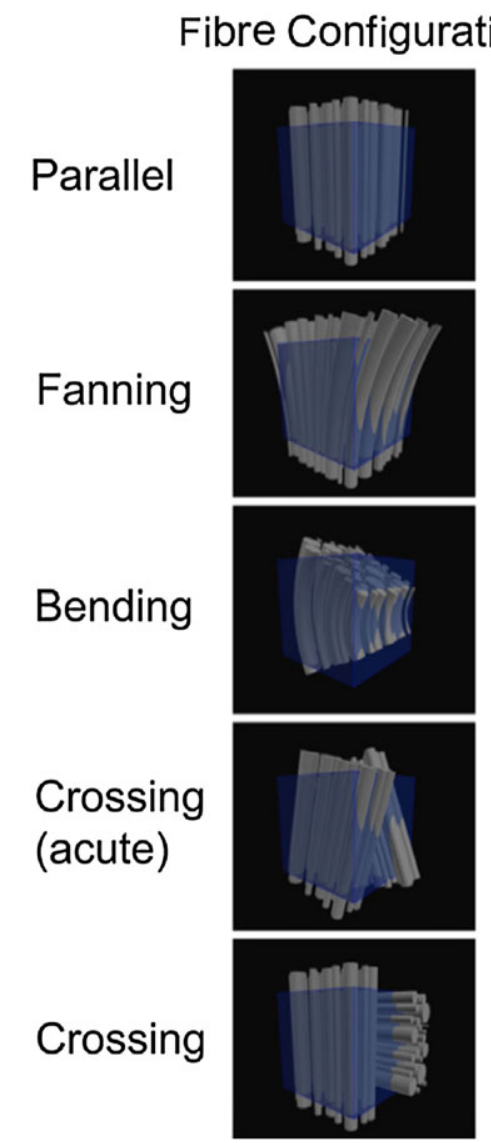

(A)

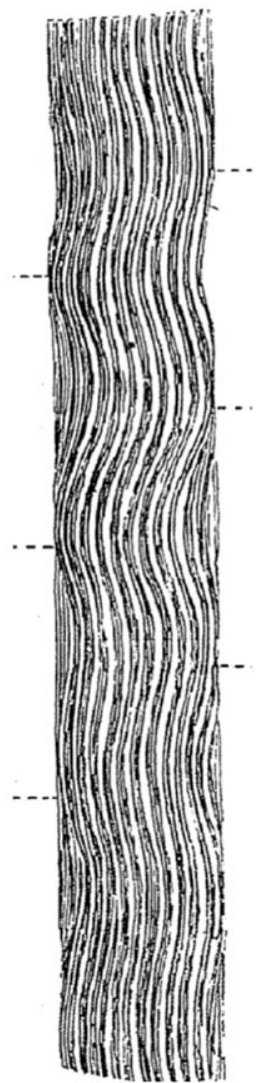

(B)

FIGURE 5 Macroscopic effects arise (A) from differences in the trajectory of multiple axons across a single voxel and (B) from non-straight axonal trajectories across a voxel. Macroscopic configurations of axons such as fanning, bending and crossing are often found in brain tissue. A from Reference 27 and B from Reference 28 are both schematic drawings. Micrographs showing axonal undulation can be found in Reference 29

(A)

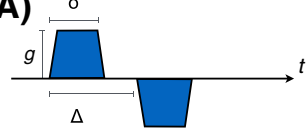

(D) $\delta$

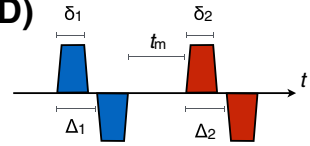

(B)

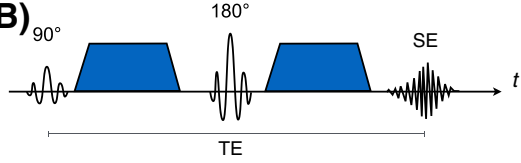

(C)

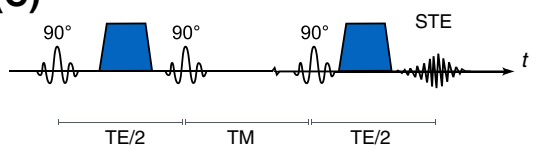

(G)

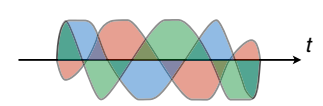

FIGURE 6 Illustration of gradient waveforms and sequences used for diffusion encoding. A, The single diffusion encoding (SDE) sequence consists of a pair of pulsed gradients defined by three parameters $\delta, \Delta$ and $g$ that together define the $b$-value. The encoding gradients must be implemented in a pulse sequence. B, The spin echo (SE) sequence is composed of an excitation pulse $\left(90^{\circ}\right)$ and a refocusing pulse $\left(180^{\circ}\right)$. C, The stimulated echo (STE) sequence replaces PGSE's $180^{\circ}$ pulse with two $90^{\circ}$ pulses that store and recall the magnetization. The effective sign of the gradient is reversed by the $180^{\circ}$ pulse in PGSE or by the last two $180^{\circ}$ pulses in the PGSTE sequence, explaining the difference between the effective waveform in $A$ and the actual waveform in B and C. Panels A,D-G show the effective waveform. D, The double diffusion encoding (DDE) sequence has two pairs of pulsed gradients, separated by a mixing time $t_{m}$. The gradient directions of the two blocks may differ. Oscillating gradients, with either smooth (E) or square (F) waveforms, can provide short encoding times when performed with high frequency $f$. G, Irregular gradient waveforms can also be used, for example, to obtain isotropic diffusion encoding

\subsection{1 | Single diffusion encoding (SDE)}

SDE sensitizes the MR signal to diffusion using a pair of gradient pulses (Figure 6A), which encode and decode the positions of spins. The sequence maintains a magnetic field gradient, defined by the gradient vector $g$, during each pulse of length $\delta$. The onsets of the two pulses have separation $\Delta$, which determines the diffusion time. Diffusion during and between the pulses leads to an attenuation of the MR signal, and this attenuation increases (i.e. signal decreases) monotonically with the variation in the distance traversed by the spins, i.e. their dispersion, between the two pulses. 
The mechanism that attenuates the signal is phase dispersion: the phase of each spin corresponds to the distance it moves in the direction of the gradient; a wider range of distances (i.e. greater dispersion) leads to a wider range of magnetization phases contributing to the signal; with greater phase dispersion, the magnetizations have a lower sum, so we observe greater attenuation of the net signal.

For free diffusion, the diffusion coefficient alone determines the range of distances for a particular $\delta$ and $\Delta$. The degree of attenuation thus provides a direct estimate of the diffusion coefficient. However, in restricted diffusion, the maximal distance any spin can travel between encoding and decoding is limited, which in turn limits the signal attenuation. The attenuation depends on the restriction distance and, in multiple dimensions, the shape of a restricting pore. Thus, multiple SDE (or other) measurements obtained by varying the different parameters of the sequence, i.e. $\delta, \Delta$ and $g$, inform estimates of size and shape of restricting pores; see References 68-70 for examples.

The $b$-value summarizes the overall diffusion weighting of a sequence and, for the SDE sequence, $b=(\gamma \delta|g|)^{2}\left(\Delta-\frac{\delta}{3}\right)$, where $\gamma$ is the gyromagnetic ratio. This formula assumes negligible ramp time in the pulses. For free diffusion, the $b$-value alone determines the signal attenuation, even though different combinations of $|g|, \delta$ and $\Delta$ can give the same $b$. Specifically, $S(b)=S(0) \exp (-b d)$, where $S$ is the signal and $d$ is the diffusivity. However, in the presence of restricted or hindered diffusion the attenuation depends separately on $|g|, \delta$ and $\Delta$. Other variables of diffusion encoding sequences include the echo time $\left(T_{\mathrm{E}}\right)$ and repetition time $\left(T_{\mathrm{R}}\right)$. These are often fixed in measurement protocols with multiple sequence-parameter combinations to ensure constant relaxation weighting.

Some unconventional implementations of SDE can offer distinct practical benefits. For example, the level of eddy currents can be reduced by implementing SDE in a double spin-echo sequence or with asymmetric gradients. ${ }^{71,72}$ While SDE is often implemented as a pulsed gradient spinecho (PGSE) sequence (Figure 6B), the pulsed-gradient stimulated-echo sequence (PGSTE) can provide longer diffusion times than standard PGSE (Figure 6C). In PGSE, the diffusion time is limited by $T_{2}$ relaxation, since the SNR decays with $T_{\mathrm{E}}$ as exp $\left(-T_{\mathrm{E}} / T_{2}\right)$. PGSTE comprises three $90^{\circ}$ pulses (rather than one $90^{\circ}$ and one $180^{\circ}$ pulse as in PGSE) that excite, store and recall the magnetization ${ }^{73,74}$ (compare Figure 6, Panels B and C). In PGSTE, only $T_{1}$ relaxation, which is slower than the $T_{2}$ relaxation pertinent in PGSE, takes place between the second and third $90^{\circ}$ pulses; PGSTE thus retains more signal at longer diffusion times. However, in PGSTE half of the signal is lost in the storage and recall process compared with PGSE. ${ }^{75}$ Thus, PGSTE has an SNR advantage over PGSE only when the time between the gradient pulses exceeds $\ln (1 / 2)\left(T_{1}{ }^{-1}-T_{2}{ }^{-1}\right)$, where $T_{1}$ and $T_{2}$ are the longitudinal and transversal relaxation times (disregarding imperfections of the RF pulses). In general in PGSTE, care must be taken to account for both the $T_{1}$ weighting and diffusion weighting from crusher and imaging gradients, which can confound both experiment design and subsequent analysis. ${ }^{76}$ Another unconventional implementation of SDE has gradient pulses of different lengths-the 'long-short' sequence, which provides particular sensitivity to pore shape. ${ }^{77}$

Common targets for SDE acquisitions in the brain are, in addition to cell size and shape mentioned above, properties such as anisotropy, fibre density, exchange and IVIM. Quantification of anisotropic diffusion for fibre direction estimation and tractography is covered elsewhere in this special issue. The degree of anisotropy of distinct compartments, such as axons, and the density of these compartments, can be estimated from experiments with multiple diffusion weightings; see, e.g., References 78 and 79. Compartment sizes, e.g. axon diameters, can be probed by SDE with variable diffusion times. ${ }^{80,81}$ Restriction manifests as a reduced signal attenuation compared with free diffusion as the diffusion time increases, and the smaller the compartment the more marked the reduction of attenuation. Exchange between compartments ${ }^{82,83}$ manifests as increased signal attenuation, compared with full restriction, as diffusion time increases. The similar effects of increasing restriction length and exchange on the signal amplitude make them challenging to disentangle in practice with SDE. ${ }^{84}$ Finally, for low $b$-values the SDE signal also captures effects of pseudorandom flow (the IVIM effect), ${ }^{65}$ which can inform on capillary blood volumes, although quantification can be sensitive to noise. ${ }^{85}$

\subsection{2 | Alternative waveforms}

Double diffusion encoding (DDE) (Figure 6D) consists of two successive SDE blocks, separated by a so-called mixing time. ${ }^{80,86}$ DDE has also been referred to as the double pulsed-field gradient spin-echo sequence, ${ }^{87}$ or the double wave-vector experiment. ${ }^{88}$ Five distinct usages of DDE target various microstructural features in different ways. ${ }^{67}$ The first varies the relative gradient directions of the two SDE blocks to quantify microscopic anisotropy (see, e.g., References 89-92). The second utilizes parallel gradients but a variable mixing time to measure exchange rates (see, e.g., References 23, 70 and 93-95). In this experiment, the first encoding block perturbs the signal fractions of different components, which then gradually restore to equilibrium. Exchange is measured by gradually increasing the mixing times while using the second encoding block to monitor this equilibration process. The third usage of DDE employs parallel and antiparallel gradients and a short mixing time to vary the degree of flow compensation and thereby improve estimation of blood volumes (see, e.g., References 85, 96 and 97). The fourth uses parallel and antiparallel gradients and short mixing time to estimate compartment sizes (see, e.g., References 88, and 98). Finally, the fifth usage targets pore size and shape distributions in heterogeneous media by noting the retention of diffusion diffraction patterns. ${ }^{99-102}$

Oscillating diffusion encoding (ODE) can be achieved with the oscillating gradient spin echo (OGSE) sequence, which replaces the constant gradient pulses in SDE with pulses that have oscillating gradient amplitude. ${ }^{103}$ Oscillating waveforms may follow smooth sine or cosine functions (Figure 6E), ${ }^{103,104}$ from square waves (Figure 6F) ${ }^{103,105,106}$ or even irregular square waves. ${ }^{107}$ With SDE, estimation of the diffusivity in small compartments requires short diffusion times, which limits the achievable $b$-value and thus the sensitivity to microscopic features. ODE can maintain $b$-value at short diffusion times by repeating multiple pulses. This can enhance the sensitivity to the diffusion coefficient in small pores and thus facilitate estimation of small sizes. ${ }^{108,109}$ However, recent work ${ }^{110,111}$ suggests that the primary benefit of ODE for the estimation of axon diameters (or cylindrical pores in general) arises in the presence of orientation dispersion or uncertainty, because ODE retains sensitivity to size 
while avoiding high $b$-values, which lead to low signal from free diffusion along cylinders that are not perfectly perpendicular to the encoding gradients.

Although SDE, DDE and ODE have been most commonly used to date, there are no theoretical reasons for limiting the gradient waveform to such designs. Benefits may arise from using irregular waveforms ${ }^{112,113}$ (Figure 6G). Specific examples include the combination of ODE with DDE into a double oscillating diffusion encoding (DODE) sequence, which may improve size and shape estimation. ${ }^{114}$ Other approaches utilize multidimensional waveforms to disentangle microscopic anisotropy from variation in isotropic diffusion, which is not possible with SDE alone. ${ }^{115}$ Examples include triple diffusion encoding (TDE), ${ }^{116}$ circularly polarized gradients, ${ }^{117}$ magic angle spinning of the $q$-vector ${ }^{118,119}$ and $q$-trajectory encoding (QTE). ${ }^{120,121}$

\section{3 | STATE OF THE ART}

This section summarizes the current state of the art in models relating the diffusion MR signal to features of brain tissue through a historical review. It then reviews current applications in biomedicine of imaging techniques based on these models.

\section{1 | Models for quantitative diffusion MRI}

Using biophysical models of diffusion to estimate tissue microstructure follows a longstanding tradition in the field of physical chemistry which applies models of this kind to determine microstructure of inanimate samples. ${ }^{122}$ For example, Packer and Rees ${ }^{81}$ quantified the size distribution of oil droplets using a model of spheres with log-normal distributed radii. Pioneering works such as this have inspired the adoption of this approach in biomedical imaging. In the early days of diffusion tensor imaging (DTI) ${ }^{123}$ it was hoped that simple indices such as the eigenvalues of the diffusion tensor, or combinations of them such as the mean diffusivity or fractional anisotropy, would reflect WM tissue properties such as myelination or fibre density. In regions of approximately parallel fibres, such as the CC, contrast in these parameters may arise from such tissue properties and has been used in this way in the literature, e.g. Reference 124. However, in general, the effects of orientation dispersion dominate such contrast and more sophisticated models are necessary to separate the effects. ${ }^{5}$ This section reviews such models in the context of assessing brain tissue microstructure.

In this article, we focus on models that exhibit the following two key features. First, the models consider the signal in a voxel as the sum of contributions from several compartments. Each compartment is posited to correspond to certain cellular components and exhibit a distinct pattern of diffusion. Often known as compartment models, they provide a natural way to describe the heterogeneity within a voxel and a mechanism to infer compartment-specific properties. This is in contrast to signal models, such as DTI, diffusion kurtosis imaging (DKI), ${ }^{125} q$-space imaging, ${ }^{80,126}$ diffusion spectrum imaging (DSI) ${ }^{127}$ and mean apparent propagator (MAP)-MRI ${ }^{128}$ for SDE, and extensions to DDE, ${ }^{15,91,129-131}$ which effectively treat each voxel as a single homogeneous compartment, thus providing only a composite view. Such signal models are reviewed elsewhere in this special issue. $^{22}$ Second, the models relate the signal directly to salient microscopic features of each compartment, typically by modelling them as simple, idealized, geometric objects. For example, models often represent axons as cylinders. Such simple geometries provide close analytical or simple numerical approximations of the signal, which enable estimation of specific microscopic features via numerical fitting. This is in contrast to, for example, bi-exponential decay models, ${ }^{12,132}$ which, although compartmental in nature, do not explicitly associate diffusion characteristics with microstructural features.

The first compartment model of neuronal tissue, pioneered by Stanisz et al., ${ }^{69}$ models nerve-tissue microstructure. It designates individual compartments for glial cells, axons and extracellular space, and aims to estimate the volume fraction of each compartment and the spatial dimensions of the cells. The glial cells are represented as identical spheres and the axons as identical prolate ellipsoids, giving rise to restricted diffusion characterized by their respective geometry. The diffusion in the extracellular space, hindered by the presence of glial cells and axons, is approximated with a tortuosity model. Tortuosity refers to the reduction of the apparent diffusivity, relative to the bulk diffusivity, in an environment with hindrance, i.e. obstacles that increase the path length of a diffusing particle. ${ }^{133-136}$ Evidence suggests this is a key factor determining the particle mobility in biological tissue. ${ }^{136}$ Stanisz et al. employ standard approximations of the tortuosity factor (see, e.g., Reference 137), for simple geometries that relate packing density to reduced apparent diffusivity as a function of the volume fractions of impeding objects-higher volume fraction leads to lower extracellular diffusivity. The model additionally accounts for the exchange of water between the intracellular compartments and the extracellular space, via the Kärger model, ${ }^{138}$ enabling estimation of the exchange rate.

More recent models of WM microstructure represent axons as straight, impermeable, cylinders. The ball-and-stick model ${ }^{139}$ represents the axons as parallel cylinders of zero radius (the 'stick'), so that water diffuses only along the cylinder axis, and dispersion in the extra-axonal space follows an isotropic diffusion tensor model (the 'ball'). The model assumes that the intra- and extra-axonal spaces have a common intrinsic diffusivity. The later and more general composite hindered and restricted models of diffusion (CHARMED) ${ }^{140,141}$ model assumes cylinders to have radii following a gamma distribution ${ }^{20}$ and to form one or more (crossing) bundles with distinct orientations. The intra-axonal signal is determined anaIytically by the cylinder radius and the intrinsic diffusivities parallel and perpendicular to the cylinder. The extra-axonal diffusion is modelled with a general diffusion tensor unconstrained by any tortuosity model. In References 142 and 143, this two-compartment CHARMED model is simplified, 
by adopting the stick model of axons, to explain the DKI metrics. In Reference 10 , the model is extended with a free water compartment ${ }^{144}$ to account for in vivo CSF contamination.

The AxCaliber technique ${ }^{10,20}$ uses the CHARMED model to estimate the ADD (axon diameter distribution). AxCaliber requires a priori knowledge of the axon fibre orientation. To enable orientation invariant mapping of axon diameter, the ActiveAx technique ${ }^{11,145}$ combines and simplifies features of Stanisz's model ${ }^{69}$ and the CHARMED model in Reference 10 to produce the minimal model of white matter diffusion (MMWMD). ${ }^{21}$ The key simplifications are a single cylindrical axon radius, a single fixed intrinsic diffusivity for the intra- and extra-axonal compartments, and modelling the apparent perpendicular diffusivity of the extra-axonal diffusion tensor using a simple tortuosity model from Reference 137. The MMWMD includes an isotropically restricted compartment (the 'Dot' compartment), similar to the glial-cell model in Reference 69, to account for unattenuating signal observed at high $b$-value in ex vivo data. Later, Panagiotaki et al. ${ }^{146}$ and more recently Ferizi et al. ${ }^{147}$ constructed a taxonomy of compartment models for WM including those above and a range of intermediate and additional compartment combinations.

Parallel cylinders assumed in the CHARMED and MMWMD models do not account for complex axonal configurations, such as bending and fanning fibres, that are widespread in WM-see Figure 5 and surrounding text. Spherical deconvolution ${ }^{8,78,148}$ aims to recover the distribution of fibre orientations, which partially captures this orientational complexity. Such techniques provide no information on fibre composition whereas generalizations of microstructural models discussed earlier potentially capture both. The ball-and-stick model generalizes straightforwardly to accommodate fibre crossing ${ }^{149}$ and orientation dispersion ${ }^{150}$ by incorporating multiple discrete sticks or a continuous distribution of sticks, respectively. Similarly, the MMWMD model generalizes to capture fibre orientation dispersion ${ }^{151}$ and crossing, ${ }^{152}$ and AxCaliber3D extends ADD mapping to crossing fibres. ${ }^{153}$

Models of complex orientation distribution led to the emergence of unified models that aim to represent both WM and GM. Jespersen et al. ${ }^{79}$ first exploit this opportunity with an analytical two-compartment model of neurites (dendrites and axons). This model, to represent highly dispersed dendritic trees, assumes that the cylinders modelling neurites follow an arbitrary orientation distribution described using spherical harmonics. Additionally, unlike the CHARMED model, the geometry of individual cylinders is not explicitly modelled. Instead, an apparent transverse diffusion coefficient is used to reflect the combined effect of cylinder radius, bending, undulation, non-vanishing permeability etc. Similar to the CHARMED model, the extra-neurite diffusion is modelled with a general diffusion tensor.

A simpler neurite model is subsequently proposed for neurite orientation dispersion and density imaging (NODDI). ${ }^{5}$ NODDI simplifies the orientation-dispersed MMWMD model ${ }^{151}$ by representing neurites as sticks rather than cylinders. The main differences from Jespersen's model in Reference 79 are a parametric description of orientation dispersion with a Watson distribution, which models isotropic orientation dispersion about a single main fibre orientation, a single fixed intrinsic diffusivity for the intra- and extra-neurite compartments, and the use of a tortuosity model to specify the apparent extra-neurite diffusion tensor. The NODDI model has been recently extended to accommodate more general orientation distributions and to relax many of its constraints on diffusivities. Tariq et al. ${ }^{154}$ replace the Watson distribution with a Bingham distribution to accommodate dispersion anisotropy. Fibre crossings are accounted for in Reference 155. Kaden et al. ${ }^{9}$ avoid any assumption on the shape of the fibre orientation distribution and further allow the estimation of the intrinsic diffusivity. However, this requires removing the CSF compartment to obtain estimates from data acquisitions currently practical for clinical studies. Jelescu et al. ${ }^{156}$ generalize this two-compartment model further, by allowing the intra- and extra-neurite compartments to have different intrinsic diffusivities and by not imposing the tortuosity model, but show that this leads to degeneracies among parameters given currently practical data acquisition. Lampinen et al. ${ }^{6}$ show that extending the data acquisition to include a spherical tensor encoding protocol yields additional information that may address this degeneracy, allowing us to relax one model constraint.

Another family of compartment models places strong emphasis on the statistical modelling of tissue heterogeneity. One of the first examples is diffusion basis spectrum imaging (DBSI). ${ }^{157}$ DBSI is unique in that it models the extra-axonal space as a spectrum of isotropic diffusion tensors. This spectrum of extra-axonal compartments is defined by an arbitrary continuous function of diffusivity that specifies the relative fraction of isotropic tensors with any given diffusivity. A similar idea is exploited in a generalization of the ball-and-stick model, ${ }^{158}$ which assumes a spectrum of intrinsic diffusivities that follows a gamma distribution. Restricted spectrum imaging ${ }^{159}$ generalizes DBSI by modelling both intra- and extra-axonal spaces in terms of diffusivity spectra. Most recently, Scherrer et al. ${ }^{160}$ have proposed a comprehensive model to capture the heterogeneity from restricted, hindered and isotropic diffusion. The heterogeneity is modelled by matrix-valued gamma distributions to generalize the similar approaches in References 158 and 161-163.

\subsection{1 | Limitations of these models}

Current models have several common limitations. First, at the heart of compartment models is the division of the measured signals into separate compartments. This is necessary to disentangle the signal into the contributions from the various underlying cellular components, but the validity of the division is difficult to assess directly. While experimental evidence suggests that the WM signal can be divided into intra- and extra-axonal origins (e.g. Reference 164), the existence of distinguishable compartments for glial cells and/or CSF has not been demonstrated explicitly. Second, the volume fractions estimated from the current models are invariably weighted by the $T_{1}$ and $T_{2}$ relaxation times of the corresponding compartment. A case in point is the absence of a myelin compartment in diffusion modelling, which is the result of the very short $T_{2}$ of myelin ${ }^{19}$ in comparison with the echo times of typical diffusion MR experiments. Third, straight, impermeable cylinders are the standard model of neurites but 
are an oversimplification. Axons are known to undulate ${ }^{63}$ and dendrites to branch ${ }^{165}$ (see Figures 4 and 5). The impermeability assumption may be reasonable in healthy WM over the typical timescales of diffusion MR experiments, but is probably violated in pathology. ${ }^{70,94,166}$ Fourth, extra-axonal space is often assumed to exhibit time-independent hindered diffusion and is modelled by a diffusion tensor, sometimes with a tortuosity model. However, in vitro experiments ${ }^{167,168}$ suggest that the extracellular space can exhibit non-Gaussian diffusion in a densely packed environment. Further studies ${ }^{169,170}$ suggest that the time dependence of extra-axonal diffusion might not be negligible for experiments involving diffusion times of about $10 \mathrm{~ms}$ to about $100 \mathrm{~ms}$. Various models assume fixed or otherwise constrained diffusivity parameters, but recent evidence $^{6,156,171,172}$ suggests violations of this assumption in the brain. Models for brain tissue often neglect the IVIM effect (see Section 2.1), which again may be reasonable for healthy tissue, but can be significantly altered in pathology.

To summarize, models potentially incorporate many biophysical influences on the signal. However, practical acquisition protocols support estimation of relatively few model parameters. Model constraints, such as ignoring certain effects, fixing certain parameters, enforcing relationships on parameter combinations or imposing prior distributions on parameter values, are unavoidable. The art of model design involves first selecting constraints that are not overly restrictive or erroneous, and second understanding the behaviour of the model when its constraints or assumptions are violated. At the time of writing, the community is far from convergence on either issue, and much debate continues over what constraints and assumptions are reasonable and how best to interpret parameters. ${ }^{6,9,156,173}$

\section{2 | Applications}

Model-based diffusion MRI aims to provide biologically more specific interpretation than standard techniques such as DTI. For example, the range of model-based approaches reviewed in the previous section aim to map, and support subsequent analysis of, indices of specific microstructural features that DTI conflates, such as neurite density, axonal diameter and neurite orientation distribution. This has great appeal for studying normal brain development, maturation and aging, as well as understanding a broad range of brain disorders. This subsection reviews current examples of such applications to biologically motivated questions.

\subsection{1 | Axonal/dendritic density and orientation distribution}

Among the first example applications, Vestergaard-Poulsen et al. ${ }^{174}$ applied Jespersen's neurite model ${ }^{79}$ to examine neurite remodelling in chronic stress studied in fixated tissue. They demonstrate a significant reduction in neurite density estimates in the hippocampus, amygdala and the prefrontal cortex. Later, Wang et al. ${ }^{175}$ adopted the same model to examine the treatment effect of marrow stromal cells, which stimulate neurite reorganization to promote functional recovery, in an animal model of traumatic brain injury. They find increased neurite density estimates in the treatment group relative to the control group and the estimated neurite density from diffusion MRI strongly correlates with histology. In both examples, ex vivo imaging is conducted following the sacrifice of the animals.

As one of the first applications involving living human subjects, Tavor et al. ${ }^{176}$ use the CHARMED model to study structural plasticity of the brain following short-term learning. They demonstrate an increase in an index of dendritic density (the fraction of the restricted compartment) in several GM areas, including hippocampus and parahippocampal gyrus, which explains the reduction in mean diffusivity (MD) observed from the corresponding DTI data. In addition, they show that the percentage change in dendritic density index induced by the learning task is consistently larger than the percentage change in MD. This example highlights the increased sensitivity for detecting subtle microscopic changes with specific markers of microstructure. However, since CHARMED is a WM model and does not explicitly model orientation dispersion, it may lead to biased estimates of dendritic density in GM and axonal density for WM with non-negligible orientation dispersion.

Kunz et al. ${ }^{177}$ apply both the NODDI and CHARMED models to assess WM microstructure in the newborn. The study demonstrates the feasibility of such analysis and shows that these biophysical models explain multi-shell data better than the diffusion tensor model. The results further suggest that NODDI's accounting for non-negligible orientation dispersion is important in all the major WM tracts, including the CC. Winston et al. ${ }^{178}$ applied NODDI to study GM microstructure for the first time. In a clinical cohort of individuals with focal cortical dysplasia, a subtype of epilepsy, the authors show that focal lesions exhibit reduced neurite density index consistent with previous histological analyses. They additionally show that the reduction in neurite density index is significantly more conspicuous than the corresponding changes in MD and FA. NODDI has since been applied to study normal brain development, ${ }^{179}$ chart the trajectory of brain maturation ${ }^{180-182}$ and investigate neurodevelopmental disorders, ${ }^{183,184}$ neurodegeneration ${ }^{185,186}$ and other neurological disorders. ${ }^{187,188}$ The simplified two-compartment CHARMED model in Reference 142 has also been increasingly adopted to enhance our understanding of brain development, ${ }^{189,190}$ neurodegeneration ${ }^{191,192}$ and neuroinflammation. ${ }^{193-195}$

\subsection{2 | Axon diameter}

Given its impact on the speed of information transfer, estimating axon diameter is of significant interest in neuroscience. Techniques such as AxCaliber ${ }^{20}$ and ActiveA $x^{11}$ are difficult on most human scanners, because the gradient strength is insufficient for sensitivity to most axon diameters ${ }^{21}$ Thus applications of these techniques are limited. Horowitz et al. ${ }^{196}$ provide the first example, applying AxCaliber, on a clinical $3 \mathrm{~T}$ system with $40 \mathrm{mT} / \mathrm{m}$ gradient, to estimate axon diameter distributions in the CC and correlate these to interhemispheric response times. However, the study has triggered debate within the community about its validity, ${ }^{197}$ given the known limitations of estimating axon diameters on systems with inadequate gradient. ${ }^{21,110,111}$ More recently, Huang et al. ${ }^{198}$ took advantage of the unique Connectom scanner with $300 \mathrm{mT} / \mathrm{m}$ gradient and used a 
simplified version of AxCaliber in a clinical cohort (multiple sclerosis) to investigate axonal pathology in the mid-sagittal section of the CC. Huang et al. ${ }^{198}$ demonstrate increased axon diameter index in the MS lesions compared with adjacent normal appearing white matter (NAWM), a finding consistent with reported histopathologic data.

\section{4 | IMPLEMENTATION AND PRACTICALITIES}

This section introduces some key steps in the implementation of practical microstructure imaging techniques and summarizes the state of the art in each.

\section{1 | Model selection}

Statistical model selection ${ }^{199,200}$ seeks the model that best explains observations. The problem is important in microstructure imaging, because our understanding of the mechanisms of MR signal generation from complex tissue architectures remains crude so we typically have to make empirical choices. Moreover, the degree of complexity that our data can support is unclear. Broadly two strategies are available.

1. Ockham's razor seeks the simplest model, i.e. the model with the smallest number of free parameters, that explains the data. This means 'explaining' in the statistical sense of fitting the data within errors expected from noise. Various information criteria, such as Akaike's information criterion (AIC) or the Bayesian information criterion (BIC), ${ }^{199}$ explicitly encode the idea by trading off model complexity against goodness of fit; Bayesian model selection ${ }^{200}$ implicitly works similarly.

2. Cross-validation ${ }^{201}$ seeks the model that best predicts unseen data. Two distinct tasks arise in predicting: (i) within-sample measurements (interpolation); and (ii) out-of-sample measurements (extrapolation).

The two strategies often broadly agree, and provably so, ${ }^{201}$ because a model that predicts unseen data best is generally one that fits visible data well but without overfitting, i.e. using the smallest possible number of parameters. However, inconsistencies certainly arise (see, e.g., Reference 147). The strongest conclusions come from using multiple model-selection strategies to identify concurrence of model ranking.

A series of publications ${ }^{146,147,202-204}$ comparing the ability of different models to explain diffusion MR signals from WM tissue, using both strategies above, provides insight into the complexity of models that such data can support. Panagiotaki et al. ${ }^{146}$ compared various models on data from fixed ex vivo rat brain tissue using the BIC. They conclude that explicit modelling of restriction in the axonal compartment, as well as isotropic restriction (as in Stanisz's glial cell compartment ${ }^{69}$ or ActiveAx's Dot compartment ${ }^{11}$ ), are essential in capturing signal variation. Ferizi et al. ${ }^{147}$ perform a similar analysis on in vivo human brain data, finding again that three compartments are necessary to capture signal variation, although the in vivo data supports less complex models than Panagiotaki's ex vivo data. Ferizi et al. ${ }^{147}$ consider AIC, BIC and cross-validation, as well as a study of the stability of model ranking over bootstrap samples, which provides useful additional insight into which conclusions are reliable. Rokem et al. ${ }^{205}$ compare the diffusion tensor model and a multi-compartment model using cross-validation with scan-rescan data. More recent work ${ }^{202-204}$ highlights the importance of fibre dispersion in modelling the signal from in vivo WM, establishing it as a key component of modern diffusion MRI models for neuroimaging. A broader study reporting results from an organized challenge across the community ${ }^{203}$ compares a wider set of model classes using cross-validation (primarily for interpolated data). Results suggest that even very crude and simple biophysical models can explain data as well as more complex signal models.

With these kinds of experiment, it is important to keep in mind that simply 'explaining' the signal, i.e. fitting measured data with few parameters, says very little about the biophysical accuracy of the model. As an illustrative example, Nilsson et al. ${ }^{206}$ conclude from studying fitting errors of various WM models that axonal permeability strongly affects the signal. However, that experiment did not consider models that incorporate the effects of orientation dispersion. Later work from the same group ${ }^{63}$ shows that orientation dispersion (a more likely biological explanation) explains the variation in the data just as well. Nevertheless, experiments that compare fitting errors and model complexity, or prediction accuracy, have great value. This value lies in the ability to reject models that cannot explain the data. This kind of statistical model selection thus provides a filter on a broad set of models we might initially brainstorm for an application, allowing us to distil a small set of candidates that are plausible explanations of what we measure. This insight is key to designing practical and reliable imaging techniques that use such models. However, empirical validation, as we review in Section 4.4, is essential to refine the choices further.

Finally, we note the importance of out-of-sample measurements in model selection (and verification). Often different or new types of measurement can reveal weaknesses or differences among models. For example, recent results from spherical tensor encoding ${ }^{6}$ or from very rich data sets with high $b$-values ${ }^{173}$ show that the NODDI model does not explain the full range of signals, particularly from grey matter, despite it fitting data from standard clinical acquisition protocols fairly well.

\section{2 | Experiment design optimization}

Experiment design refers to the finite set of data points we sample from the, generally infinite, space of possible measurements. The choice of experiment design is critical in obtaining good parameter estimates in any model-based estimation task. In quantitative MRI, the experiment design 
is the choice of pulse-sequence parameters in each image that provides the data to which we fit the model. In diffusion MRI, the pulse-sequence parameters might be, depending on the complexity of the model, just the $b$-value or a more complete set of defining parameters, e.g. $\delta, \Delta, g, T_{E}$, for the SDE sequence. ${ }^{145} \mathrm{~A}$ good design has to balance competing effects, such as increasing sensitivity with $b$-value but lower signal-to-noise ratio as $T_{E}$ increases to accommodate higher $b$-value. To design a protocol for front-line application, i.e. use in a biomedical study or clinical application rather than development, we seek the combination of measurements that, for a particular budget of acquisition time, maximizes sensitivity to the parameters of a model we have chosen to use.

The Fisher information matrix ${ }^{207} \mathrm{~F}$ is the expectation of the second derivative of the log likelihood of the measured signal. It is a key tool in experiment design optimization, because the inverse of $F$ provides an estimate of the covariance matrix (i.e. the expected uncertainty of the estimates) of the model parameters given the design. Design optimization typically seeks to minimize the variance of the parameter estimates. Thus, several scalar functions of $F$, e.g. $\operatorname{Tr}\left(F^{-1}\right), 1 / \operatorname{Det}(F)$ etc. (see References 207 and 208), provide suitable objective functions to minimize with respect to the combination of sequence settings comprising the acquisition protocol.

Applications of these ideas in quantitative MRI include Dixon imaging, ${ }^{209}$ quantitative magnetization transfer ${ }^{210}$ and arterial spin labelling. $^{211}$ In diffusion MRI microstructure imaging, Reference 145 outlines a framework for optimizing the design in this way. The acquisition protocols for ActiveAx in References 11 and 21, NODDI in Reference 5 and filter exchange imaging (FEXI) in Reference 212 all use this approach. These optimized designs can produce substantial improvements in parameter maps over ad hoc designs; see Figure 7 for an example.

Two caveats are important with this experiment design strategy.

1. For any non-linear model, $F$ depends on the choice of model parameters. This creates circularity: we can only optimize the design for predefined parameter values, yet we do not know the parameter values, which is why we estimate them. Moreover, in MRI, the parameter values vary spatially, but we can only choose one protocol common to all voxels. In practice, we break this circularity by: (i) assuming that the optimality of the design changes slowly as a function of the parameter values; and/or (ii) selecting a range of combinations of representative parameter values and averaging the objective function over each combination.

2. The optimization has high dimension. A typical diffusion MRI protocol includes of the order of 100 diffusion-weighted images. With SDE, each has at least five degrees of freedom, leading to a 500-dimensional optimization problem. ${ }^{145}$ Such problems are usually intractable. If we consider the whole gradient waveform, as in References 112, 113 and 120, the problem explodes further to hundreds of parameters per measurement. To mitigate this, we can impose constraints on the protocol to bring the dimensionality of the problem under control. For example, the framework in Reference 145 divides the DWIs into a relatively small number, $M$, of high-angular-resolution diffusion imaging (HARDI) shells each with common pulse timing. This reduces the dimensionality to $3 \mathrm{M}$. Even with this relatively small number of dimensions, the search requires multiple runs of a lengthy stochastic optimization to find good solutions, as the objective function has many local minima. An alternative strategy is to optimize proxy quantities, such as the maximal $b$-value in the minimum echo time. ${ }^{120}$

These caveats reveal that experiment design is an inexact science at least in application to microstructure imaging. However, even the suboptimal solutions we obtain can make substantial practical differences; again see Figure 7. In fact, even in very high-dimensional experiment design problems, optimization can find useful solutions and provide important insight into the choice of protocol; see, for example,

(A) Original

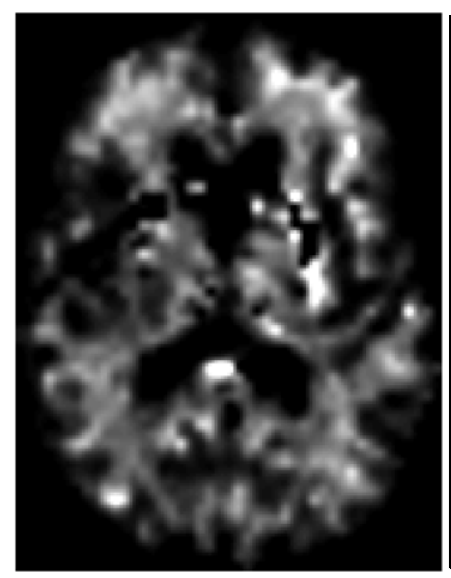

Nilsson et al (2013) 45 minutes
(B) Optimised

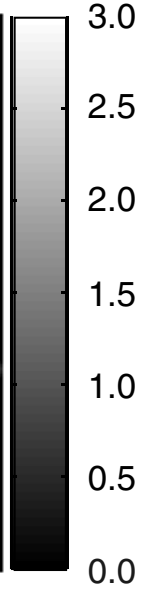

0.0

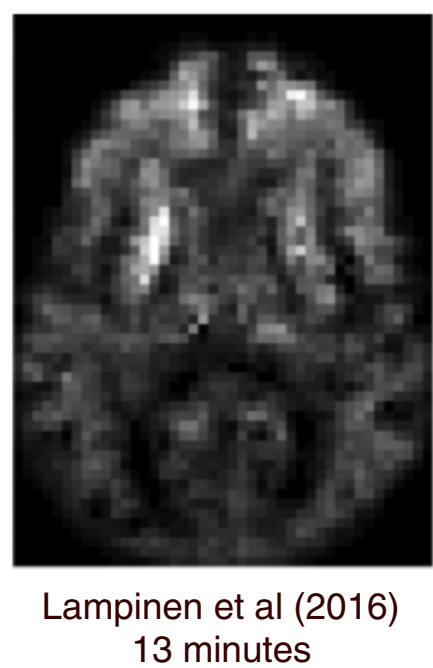

4.0

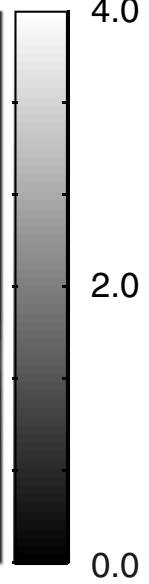

0.0 13 minutes

FIGURE 7 Comparison of AXR maps from the original ${ }^{213}$ experiment design and the optimized ${ }^{212}$ experiment design. The map on the right shows clearer structure and contrast, despite requiring only $13 \mathrm{~min}$ of acquisition as opposed to $45 \mathrm{~min}$ for the map on the left. (The slices do not exactly match, as the acquisitions come from different subjects in a different study, but they show typical contrast for each protocol.) The unit of the grey scale is $1 / \mathrm{s}$ 
Reference 113. Validation experiments using simulations, physical phantoms and relevant biological samples are important to verify efficacy of any design.

We emphasize that the experiment design strategy above is only appropriate once a suitable model has been identified. Preceding steps in the development of microstructure imaging techniques can require quite different design strategies. For example, model comparison experiments (Section 4.1) tend to cover the useful measurement space as widely as possible to reveal all significant and potentially useful effects. This contrasts strongly with F-based designs, which cluster measurements around a few points at which the signal changes rapidly with parameter values. Other developmental steps use F-based optimization, but in different ways. For example, to establish (or rule out) sensitivity of a particular family of measurement to a particular parameter (can we measure any realistic axon diameters in white matter with SDE and $40 \mathrm{mT} / \mathrm{m}$ gradients?) requires the best possible combination of measurements within the family; see, for example, Reference 145 . Similarly, to make statements about which pulsesequence family is most sensitive to a particular parameter (is OGSE more sensitive to axon diameter than SDE?) requires the optimal combination of measurements within each family; see, for example, References 110 and 214 . The literature on experiment design (e.g. Reference 208) contains a variety of other strategies that are relatively unexplored in microstructure imaging and quantitative MRI.

\section{3 | Parameter estimation}

Once we have a model and some data, a variety of options are available for fitting the model to the data to obtain parameter estimates. An accompanying article in this special issue ${ }^{22}$ focuses on some aspects of this challenge, but we summarize key techniques briefly here for completeness.

The standard procedure is to use maximum likelihood estimation, typically via non-linear fitting such as gradient descent, independently in each image voxel. Standard implementations of NODDI, AXR imaging and AxCaliber all use this approach and typically report a single best-guess parameter estimate in each image voxel, although gradient descent techniques do often provide an additional measure of confidence in each parameter estimate, which can be useful. Sampling techniques, such as Markov chain Monte Carlo (MCMC), sample the posterior distribution on the parameter values, which can provide a more complete picture of confidence in each parameter estimate as well as avoiding local minima problems associated with gradient descent; ActiveAx uses a multi-stage fitting process involving gradient descent followed by MCMC sampling. The use of averaging across direction prior to fitting, ${ }^{9,90,171,215-218}$ as in the spherical mean technique ${ }^{9,171}$ or methods that compute higher-order moments, ${ }^{173,219,220}$ can provide invariants to the fibre orientation distribution sensitive only to fibre composition. This can avoid fitting to very large numbers of measurements by non-linear optimization, which increases speed and enhances stability.

Recently, various techniques move away from gradient descent to use linearized fitting routines, convex optimization or dictionary-based methods. These can avoid local minimum problems and dramatically reduce computation time, at the cost of some precision of the final estimates. The AMICO framework, ${ }^{221}$ LEMONADE, ${ }^{222}$ WMTI, ${ }^{142}$ and Reference 223 are all examples. However, confidence estimates are less straightforward to obtain from such techniques than from classical parameter estimation.

Exploitation of spatial coherence of brain images can help to increase accuracy of parameter estimates and resolve ambiguities. In both WM and GM, the statistics of tissue composition are often similar in neighbouring voxels, suggesting that we can, sometimes, pool information from neighbouring voxels, rather than treating each independently, to better inform parameter estimates. In WM, the macroscopic continuity of axon bundles potentially provides further constraints on parameter estimates and their spatial variation. Morgan ${ }^{224}$ fitted the trend in axon diameter across the CC to all voxels in the region; Scherrer et al. ${ }^{160}$ used the BOBYQA algorithm ${ }^{225}$ to exploit spatial coherence during fitting of the DIAMOND model. Recent combinations of global tractography and microstructural parameter estimation ${ }^{226,227}$ also embrace this idea.

\subsection{Empirical evaluation}

In order to assess the degree to which model parameters capture the underlying features of the tissue, evaluations can be performed by simulations or by combined dMRI and microscopy measurements in phantoms or tissues. Figure 8 summarizes the spectrum of approaches. Each provides a different balance between realism of microstructure and control of ground-truth values. Numerical approaches typically offer high control at the expense of realism, whereas the reverse holds true for measurements in tissue.

Numerical simulations support investigations of the robustness of parameter estimates under ideal conditions. The basic premise is to predict the signal for a given measurement protocol, add noise and fit the model in multiple repetitions. Synthesizing from and fitting back the same model can establish effects of protocol design and noise level on parameter estimates (see, e.g., Reference 231), the range in which parameters can be estimated with high accuracy ${ }^{84,232}$ and the interplay between the hardware constraints and parameter estimates, e.g. the available gradient strength and the estimated axon diameter index, as in Reference 21. Such evaluations establish an upper bound for the parameter accuracy, which can be compared across sampling protocols.

We can learn how model parameters respond under departures from the model assumptions by generating data using a model or procedure that is more complex than the one used for fitting. Numerical approaches are useful in this context, and have been used to illuminate how DTI parameters respond to e.g. crossing fibres or partial volume effects, ${ }^{14,233,234}$ and to study degeneracy in parameter estimation. ${ }^{156}$ Monte Carlo (MC) simulations are especially useful to simulate dMRI of complex microstructure. ${ }^{84,228,235,236}$ In $\mathrm{MC}$ simulations, random walkers are released in a numerically defined microstructure substrate. For each walker, the phase accrued from a simulated gradient 

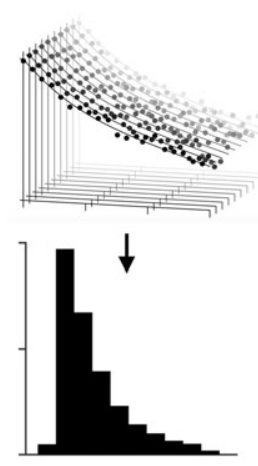

(A) Numerical analysis
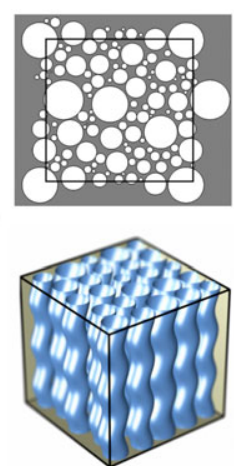

(B) Monte Carlo
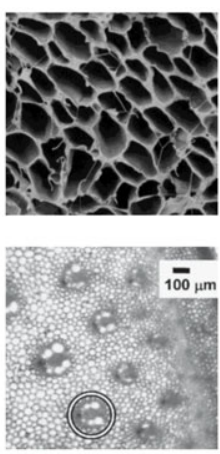

(C)Phantoms

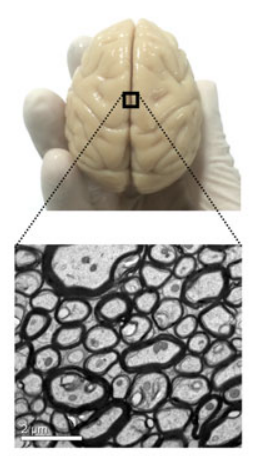

(D) Fixed tissue

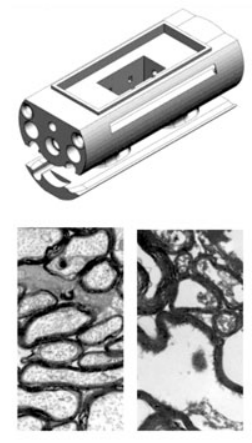

(E) Viable tissue

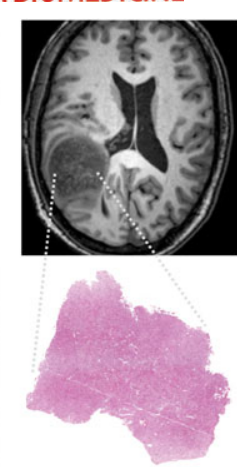

(F) In vivo + ex situ

FIGURE 8 Methods for empirical validation of microstructure imaging, showing the spectrum of techniques with increasing realism but decreasing specificity of ground truth (left to right). A, Numerical simulations can be used to simulate data (top) and evaluate the robustness of model parameter estimation (bottom). B, Monte Carlo simulations can be used to investigate diffusion in simple to complex digital substrates. ${ }^{228} \mathrm{C}$ Physical phantoms can be composed of inert material (top, from Reference 229) or of biological material (bottom, from Reference 230).

Measurements on phantoms provide an opportunity to validate the pulse sequence implementation, as well as sanity check parameter estimates in simple geometries where ground truth is relatively well defined and to some extent controllable. D, Fixed tissue offers means to obtain high-quality data without motion artefacts, while still having access to ground truth via, for example, electron microscopy (bottom, courtesy of Mark Burke). E, Measurements on viable (rather than fixed) tissue in a maintenance chamber can be performed to alleviate potential alterations of the tissue microstructure from fixation; the image pair at the bottom shows electron microscopy from tissue that spent $10 \mathrm{~h}$ in a tissue maintenance chamber (left) compared with tissue deprived of glucose and oxygen for $2 \mathrm{~h}$ (right); courtesy of Simon Richardson. F, In vivo imaging combined with ex situ microscopy can be performed in preclinical conditions or in patients undergoing surgery to assess the agreement of MRI-based and microscopybased analysis

waveform is recorded and used to predict the signal. Substrates can be constructed to match model assumptions of, for example, parallel nonabutting cylinders, as in References 84, 28, 235 and 239. Alternatively, the model can be challenged by constructing substrates with higher complexity. Such simulations have been conducted to learn how the model parameters respond to changes in fibre shape, permeability, undulation or dispersion. ${ }^{34,63,84,114,228}$ Segmented histology slides can also yield a substrate with a high level of complexity in both the intra-axonal and extracellular spaces. ${ }^{109,237}$

Physical phantoms, which represent but generally simplify the tissue of interest, provide the opportunity to test models with measured data but from idealized or simplified samples. Ground-truth values of model parameters can sometimes be controlled in the phantom construction, and importantly obtained by independent methods such as microscopy. Different materials are used for phantom construction. Inert materials such as glass or plastics offer long shelf life, high reproducibility and good control over microstructural parameters. Conversely, biological phantoms, e.g. vegetables or cell cultures, have shorter shelf life, but are often cheap and easy to prepare, although the features of the microstructure are harder to control and measure. Axon-like phantoms have been constructed from hollow glass capillaries and used to verify diffusion models, ${ }^{238,239}$ validate the relation between pore sizes and diffraction patterns from DDE, ${ }^{101}$ test size estimation with ODE, ${ }^{240}$ study microscopic anisotropy ${ }^{241}$ and test dMRI with free gradient waveforms. ${ }^{214}$ Fibre-like phantoms can also be constructed with coelectrospinning, ${ }^{229,242}$ or formed by liquid crystals. ${ }^{243}$ Asparagus stems have capillaries that can approximate large axons, ${ }^{230,237,244}$ while asparagus puree can be used to study microscopic anisotropy. ${ }^{218}$ Phantoms that approximate round cells can be constructed from oil-water emulsions. ${ }^{81}$ Such phantoms were used to test compartment models ${ }^{81,105,245}$ and later to test multimodal microstructure estimation. ${ }^{246}$ Yeast cultures also form phantoms with isotropic diffusion in distinct intra- and extracellular compartments. ${ }^{94,167,247,248}$ Yeast cells feature temperature-dependent membrane permeability, detectable by DDE, ${ }^{70}$ and cell sizes in the range of 4-8 $\mu \mathrm{m}$, which makes yeast cell suspensions an ideal testbed for methods aimed at cell size estimation. ${ }^{98,107}$

Measurements in a microstructural environment close to in vivo can be performed by using fixed tissue, which offers many of the same benefits as phantoms. A drawback is that the ground-truth microstructure cannot be controlled and may be less well characterized than in phantoms. Many challenges must be addressed to obtain high-quality data of fixed tissue. ${ }^{249}$ It is of key importance to minimize the interval between death and fixation, ${ }^{250}$ although once fixated successfully the microstructure and the diffusion parameters can be stable for years. ${ }^{249}$ However, the degree to which the fixation alters the microstructure is unknown. ${ }^{251}$ Alternatively, measurements on viable tissue samples can be obtained to avoid possible fixative-related biases. ${ }^{251-253}$ Results from comparisons between dMRI-derived parameters and microscopy of fixed tissue have revealed timedependent diffusion congruent with diffusion restricted within axon-sized compartments. ${ }^{20,68}$ OGSE-frequency dependence in both the intra-axonal and extracellular spaces, ${ }^{109}$ and good agreement of myelinated neurite fractions from dMRI and histology. ${ }^{4}$ Image analysis of fixed tissue has also been used to quantify levels of axonal orientation dispersion. ${ }^{7,254}$

Direct validation where the same tissue is used for both MRI and histology is particularly useful to guide the interpretation of model parameters, for example, by comparing axon diameters estimated from dMRI with those estimated from histology. ${ }^{10}$ Naturally, such validation is impossible in healthy human brain tissue, and can be challenging even in a preclinical setting. Indirect validation by comparison to literature values is an 
alternative, for example, regarding axon diameter estimation in humans. ${ }^{11}$ Resections in patients with e.g. brain tumours may, however, offer a rare opportunity for direct validation. ${ }^{255,256}$

\section{5 | Development pipeline}

The various steps above are all important in the development of successful microstructure imaging techniques. Figure 9 illustrates broadly how the different steps fit together. The key messages are the following.

I. The process is iterative rather than linear: earlier steps predominantly inform later steps, but, very often, later steps will reveal new information that require rethinking and repetition of earlier steps.

II. Theoretical work to understand tissue structure and signal generation are necessary to find good models, but not sufficient. Empirical steps are essential to refine candidate models that theoretical brainstorming identifies. This includes both statistical model selection to reject models that do not explain the data, and validation against independent measurements to find models that best estimate histological parameters.

III. Theoretical development of the model must go hand in hand with the design and choice of sequence and measurement protocol to ensure that the data acquired provide sensitivity to the parameters we intend to estimate.

IV. Very good arguments must be identified for increasing model complexity over the simplest available models that reasonably explain the trends in the data (as described in Section 4.3). Robustness, repeatability and computation time are extremely important for clinical and biomedical application. These requirements favour simpler models even further than statistical model comparison alone. On the other hand, the assumptions of simpler models may be violated more easily and frequently. Users must be cautious of this and have an understanding of how the model behaves when it happens. Of course, it is much easier to reason about anomalous parameter values in a model with two variables than a model with 10 , so the ability to detect and explain anomalies again should favour simple models.

V. Similarly, very good arguments must be identified for using pulse sequences that go beyond the simplest possible (e.g. SDE via PGSE). In particular, the benefits of non-standard sequences in terms of parameter sensitivity are sometimes quite subtle. At the same time, such

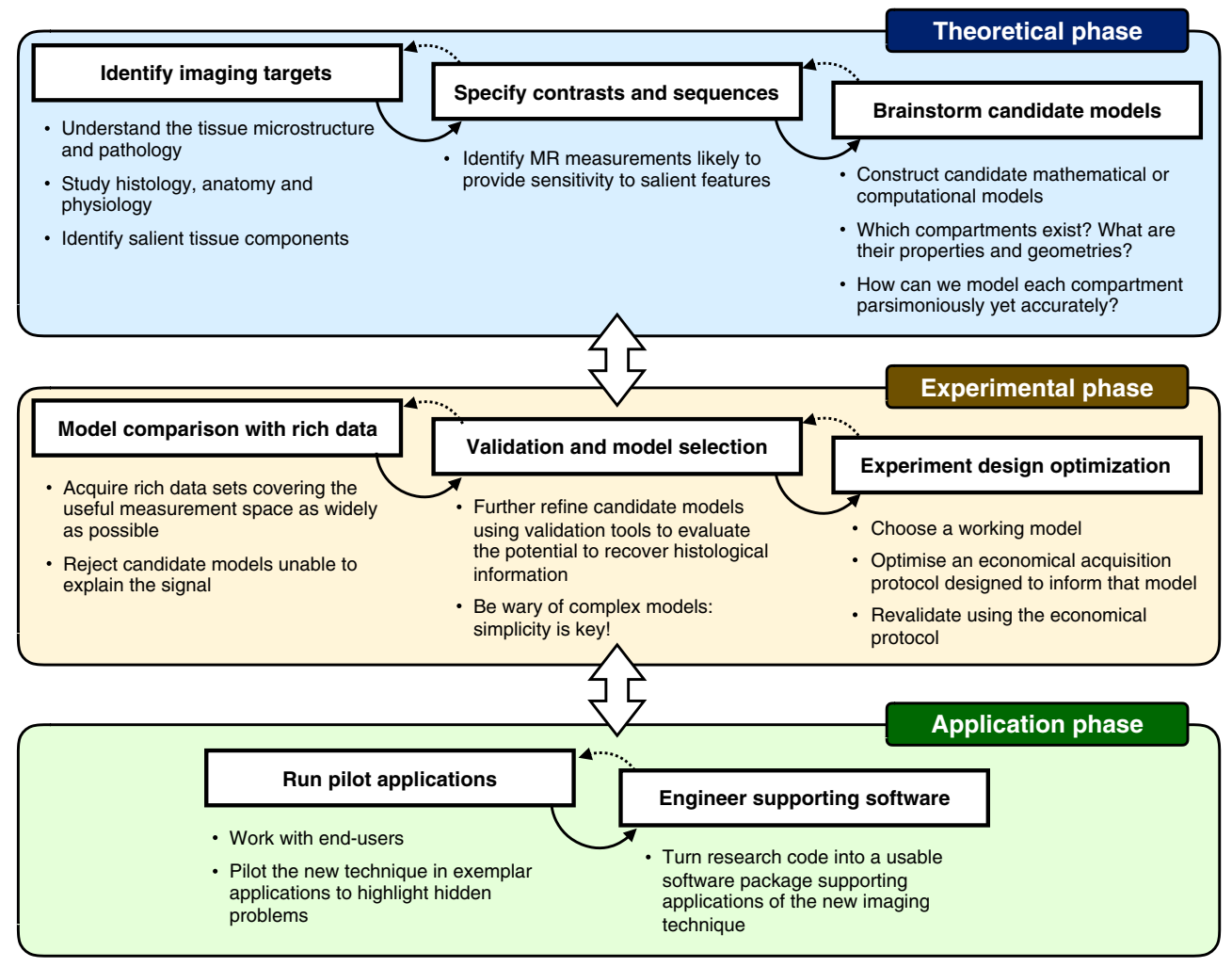

FIGURE 9 Microstructure imaging development pipeline. We identify three phases. A theoretical phase first gains an understanding of the microstructure of the tissue of interest, it then brainstorms measurements (i.e. choice of sequence and protocol) likely to provide sensitivity to the imaging targets within that tissue, and similarly brainstorms candidate models linking these measurements to tissue features. An experimental phase then acquires data to test, compare, validate and select working models and acquisition protocols. Finally, an application phase develops user-friendly software enabling widespread uptake of the technique. This must involve work with end-users in real-world applications to turn prototype code into a working system, understand user behaviour and provide the necessary education to use the technique appropriately. The phases, as well as the steps within them, are mutually informative, so the pipeline is iterative rather than linear 
measurements may incur unexpected and easily missed artefacts. Benefits and drawbacks should thus be analysed and tested carefully. Moreover, lack of availability on commercial MRI scanners can hinder wide uptake even when benefits are clear.

VI. Robust and usable software together with exemplar applications are essential for translation to widespread uptake. Both require substantial investment of time, but are essential steps in identifying problems of usage and interpretation, as well as engineering a technique for frontline application.

\section{5 | FUTURE AND EMERGING IDEAS}

Despite the great promise, current microstructure-imaging techniques remain simplistic with clearly identifiable limitations. A variety of paths are available for amelioration of current limitations and for greater advances towards the next generation of quantitative microstructural imaging techniques.

\section{1 | Better models}

The biophysical models underpinning current microstructure imaging techniques remain crude. The practical demands for simple models, which Section 4.5 summarizes, necessitate major simplifying assumptions, such as fixed diffusivity parameters, fixed axon diameter distribution, no exchange between compartments, perfectly straight and cylindrical axons, simplistic tortuosity approximation, ... the list goes on. A variety of anomalous results point to the fact that these design choices are oversimplifications. For example, diffusion MRI axon diameter mapping techniques typically overestimate the axon diameter in comparison to classical histology by a factor of 3 or more ${ }^{11,21,151}$; isotropic diffusion encodings suggest similar mean diffusivity in the intra- and extracellular spaces, ${ }^{121}$ which is incompatible with diffusion models such as NODDI, at least in GM; measurements at high $b$-value may not support tortuosity constraints, ${ }^{173}$ although the intuition that greater axon density reduces perpendicular diffusivity seems reasonable, so the result perhaps suggests other model inaccuracies. Current models neglect effects such as exchange, axonal undulation, branching of dendrites and glial cell processes. Simply adding additional model parameters to capture such effects leads to over-parametrization preventing any sensible parameter estimates. However, independent experiments to measure these various quantities potentially enable strong priors to help account for such effects. Recent trends toward computational rather than mathematical models (see, for example, 166 and 167), can help capture effects, such as undulation, exchange, branching and extracellular tortuosity, for which mathematical models are intractable. They do not help with over-parametrization, but such models can help identify the locus of possible parameter combinations that can explain measurements thus supporting more reasoned downstream inference that accounts for parameter uncertainty.

\section{2 | Advanced pulse sequences}

Current techniques in widespread application use the standard SDE sequence. However, as discussed in Section 2.1, advanced pulse sequences potentially offer greater sensitivity to tissue features we currently estimate as well as additional sensitivity to new features. Research remains to characterize fully the benefits of different sequences and the precise situations in which these benefits arise, although certain specific benefits are already clear. For example, OGSE enhances sensitivity to axon diameter in simple compartment models in the presence of orientation dispersion or uncertainty ${ }^{110}$; DDE and $q$-space trajectory imaging can discriminate microscopic anisotropy from distributed microscopic pore size, which SDE cannot distinguish $90,91,115,218,256$; DDE can enhance sensitivity to exchange ${ }^{23,94}$ and improve estimation of blood volumes. ${ }^{85}$ Several challenges remain in exploiting the potential benefits of such sequences: (i) making them readily available and stable on standard clinical platforms; (ii) optimizing acquisition strategies and imaging protocols to yield acceptable scan times; (iii) identifying specific applications in which they offer a clear benefit; and (iv) constructing sufficiently stable and parsimonious models that relate their signals to useful tissue features. Immediate applications do arise in verifying, or highlighting limitations of, models designed using only standard SDE sequences, as in Reference 6.

\section{3 | Parameter estimation}

We can expect methods of parameter estimation to become more reliable, enhancing the precision of parameter maps. The dictionary-based approaches discussed in Section 4.3 offer great promise and are essential for processing very large databases of images. Techniques such as multi-model inference ${ }^{199}$ are largely unexplored in microstructure imaging, but should help to avoid consistent bias in parameter estimates while avoiding over-fitting. Such techniques also enable quantification of uncertainty in parameter estimates arising not just from noise, but also from uncertainty in the choice of model, thus quantifying more precisely our belief that parameter estimates reflect true underlying tissue microstructure. 


\subsection{New hardware}

Next-generation MRI scanners can dramatically enhance the performance of microstructure imaging techniques. Stronger magnetic field gradients have major benefit by: (i) increasing the signal to noise of diffusion-weighted measurements by reducing the echo time required for a particular $b$ value; (ii) reducing the lower bound on the pore size to which the signal is sensitive. Dyrby et al. ${ }^{21}$ demonstrate the benefits of enhanced gradient strength on estimation and mapping of axon diameter in fixed monkey brains, confirming earlier simulation work such as Reference 230 . The ex vivo work in Reference 12 exploits $50 \mathrm{~T} / \mathrm{m}$ gradients. Recent work on the Boston Connectom scanner ${ }^{257}$ shows promising results in axon diameter mapping in live subjects both in health (see, e.g., 13) and in disease. ${ }^{198}$

Higher field strength also offers advantages for diffusion MRI and microstructure imaging in general by providing images with higher signal and higher spatial resolution. Although higher field strength also reduces $T_{2}$ potentially to the detriment of diffusion MR signals, recent work ${ }^{258,259}$ confirms benefits of moving to higher field as well as the complementarity of $3 \mathrm{~T}$ and $7 \mathrm{~T}$ data, which can combine advantages of higher spatial resolution at high field with higher signal at high $b$-value available at lower field. ${ }^{260}$

\section{5 | Multimodality}

A major new avenue for microstructure imaging in the future is to combine information from diffusion MRI with that from other MR contrasts or even other modalities. Various other quantitative MR techniques offer complementary information on tissue microstructure to diffusion MRI, and the construction of models informed by multiple contrasts has the potential to resolve ambiguities that are intrinsic to single-modality approaches. The first multi-modality microstructure imaging techniques are just starting to appear. For example, recent work on g-ratio estimation and mapping combines estimates of myelination from quantitative magnetization transfer ${ }^{124,261}$ or myelin water imaging ${ }^{262}$ with estimates of axonal density from diffusion MRI, albeit without using an integrated model of both contributing modalities. Early work combining estimates of pore density from diffusion MR and optical imaging provides an example of an integrated model. ${ }^{246}$ Exciting possibilities arise in joint modelling of relaxometry (see, e.g., Reference 263) and susceptibility imaging (see, e.g., Reference 264), with diffusion MRI, since the former are confounded by microstructural orientation effects, which the latter is able to estimate with relatively high accuracy. However, serious challenges arise in multi-modal microstructure imaging. First, the idea demands unified models relating MR to tissue features across multiple contrasts, none of which currently have broadly agreed models even independently. Second, practical issues arise in processing and alignment of images from different contrasts, which often use quite different acquisition and reconstruction algorithms, to ensure sets of measurements from corresponding volumes of tissue.

\section{6 | Further applications}

As we mentioned in Section 3.2, the range of applications of microstructure imaging techniques in the brain is expanding rapidly. The idea further extends naturally to non-brain applications, although different models are required to explain the signal. Recent developments in non-brain cancer imaging ${ }^{265-267}$ extend the paradigm from the brain with extremely promising results. Many other opportunities are available in, for example, imaging the heart, muscle tissue, liver, kidney, lung, placenta and many other solid organs. The appetite for such techniques from clinicians and biomedical researchers can be very high, because they rely solely on painstaking histological analysis for specific information on tissue structure. However, it can take time to educate such users in the precise capabilities of such techniques. As non-invasive approaches become more available and popular, we must be careful to understand their limitations and educate users of such limitations to avoid misinterpretation. Nevertheless, exposing techniques to users is essential to identify both potential and limitations-we hope an understanding of the pipeline outlined in Figure 9 can help to balance these competing pressures and expedite the development of powerful future microstructure imaging tools.

\section{ACKNOWLEDGEMENTS}

EPSRC grants EP/N018702/1 and EP/M020533/1 fund DCA's and HZ's work on this topic. The Swedish Foundation for Strategic Research (grant no AM13-0090) and the Swedish Research Council (grant no 2016-03443) fund MN's work on this topic. The Capital Region Research Foundation for Healthcare (grant no A5657) and the Danish Multiple Sclerosis Foundation (grant no A31910) fund TBD's work on this topic.

\section{ORCID}

Daniel C. Alexander (10 http://orcid.org/0000-0003-2439-350X

Tim B. Dyrby (D) http://orcid.org/0000-0003-3361-9734

Markus Nilsson (10) http://orcid.org/0000-0002-3140-8223

Hui Zhang (1) http://orcid.org/0000-0002-5426-2140

\section{REFERENCES}

1. Brodmann K. Vergleichende Lokalisationslehre der Grosshirnrinde. Leipzig: Johann Ambrosius Barth; 1909.

2. Ramon y Cajal S. Les Nouvelles Idées sur la Structure du Système Nerveux chez l'Homme et chez les Vertébrés. Reinwald; 1894.

3. Braak H, Braak E. Staging of Alzheimer's disease-related neurofibrillary changes. Neurobiol Aging. 1995;16:271-278. discussion 278-284 
4. Jespersen SN, Bjarkam CR, Nyengaard JR, et al. Neurite density from magnetic resonance diffusion measurements at ultrahigh field: comparison with light microscopy and electron microscopy. Neuroimage. 2010;49:205-216.

5. Zhang H, Schneider T, Wheeler-Kingshott CA, Alexander DC. NODDI: practical in vivo neurite orientation dispersion and density imaging of the human brain. Neuroimage. 2012;61:1000-1016.

6. Lampinen B, Szczepankiewicz F, Martensson J, van Westen D, Sundgren PC, Nilsson M. Neurite density imaging versus imaging of microscopic anisotropy in diffusion MRI: a model comparison using spherical tensor encoding. Neuroimage. 2017;147:517-531.

7. Leergaard TB, White NS, de Crespigny A, et al. Quantitative histological validation of diffusion MRI fiber orientation distributions in the rat brain. PLoS ONE. 2010;5: e8595

8. Tournier JD, Calamante F, Gadian DG, Connelly A. Direct estimation of the fiber orientation density function from diffusion-weighted MRI data using spherical deconvolution. Neuroimage. 2004;23:1176-1185.

9. Kaden E, Kelm ND, Carson RP. Does MD, Alexander DC. Multi-compartment microscopic diffusion imaging. Neuroimage. 2016;139:346-359.

10. Barazany D, Basser PJ, Assaf Y. In vivo measurement of axon diameter distribution in the corpus callosum of rat brain. Brain. 2009;132:1210-1220.

11. Alexander DC, Hubbard PL, Hall MG, et al. Orientationally invariant indices of axon diameter and density from diffusion MRI. Neuroimage. 2010;52:1374-1389.

12. Ong $\mathrm{HH}$, Wehrli FW. Quantifying axon diameter and intra-cellular volume fraction in excised mouse spinal cord with $q$-space imaging. Neuroimage. 2010;51:1360-1366.

13. Duval T, McNab JA, Setsompop K, et al. vivo mapping of human spinal cord microstructure at $300 \mathrm{mT} / \mathrm{m}$. Neuroimage. 2015;118:494-507.

14. Szczepankiewicz F, Lasic S, van Westen D, et al. Quantification of microscopic diffusion anisotropy disentangles effects of orientation dispersion from microstructure: applications in healthy volunteers and in brain tumors. Neuroimage. 2015;104:241-252.

15. Shemesh N, Barazany D, Sadan O, et al. Mapping apparent eccentricity and residual ensemble anisotropy in the gray matter using angular doublepulsed-field-gradient MRI. Magn Reson Med. 2012;68:794-806.

16. Lawrenz M, Finsterbusch J. Double-wave-vector diffusion-weighted imaging reveals microscopic diffusion anisotropy in the living human brain. Magn Reson Med. 2013;69:1072-1082.

17. Laule C, Kozlowski P, Leung E, Li DK, Mackay AL, Moore GR. Myelin water imaging of multiple sclerosis at 7 T: correlations with histopathology. Neuroimage. 2008;40:1575-1580.

18. Deoni SC, Mercure E, Blasi A, et al. Mapping infant brain myelination with magnetic resonance imaging. J Neurosci. 2011;31:784-791.

19. Whittall KP, MacKay AL, Graeb DA, Nugent RA, Li DK, Paty DW. In vivo measurement of $T_{2}$ distributions and water contents in normal human brain. Magn Reson Med. 1997;37:34-43.

20. Assaf Y, Blumenfeld-Katzir T, Yovel Y, Basser PJ. AxCaliber: a method for measuring axon diameter distribution from diffusion MRI. Magn Reson Med. 2008;59:1347-1354.

21. Dyrby TB, Sogaard LV, Hall MG, Ptito M, Alexander DC. Contrast and stability of the axon diameter index from microstructure imaging with diffusion MRI. Magn Reson Med. 2013;70:711-721.

22. Novikov D, Jespersen SN, Kiselev VG, Fieremans E. Quantifying brain microstructure with diffusion MRI: theory and parameter estimation. ArXiv Preprint arXiv. 2016;1612:02059

23. Nilsson M, van Westen D, Stahlberg F, Sundgren PC, Latt J. The role of tissue microstructure and water exchange in biophysical modelling of diffusion in white matter. Magn Reson Mater Phys Biol Med. 2013;26:345-370.

24. Buzsaki G, Logothetis N, Singer W. Scaling brain size, keeping timing: evolutionary preservation of brain rhythms. Neuron. 2013;80:751-764.

25. Fiala JC, Spacek J, Harris KM. Dendrite structure. In: Stuart G, Sprutson N, Hausser M, eds. Dendrites. Oxford: Oxford University Press; 2007.

26. Zaqout S, Kaindl AM. Golgi-Cox staining step by step. Front Neuroanat. 2016;10:38.

27. Seunarine KK, Alexander DC. Multiple fibres: beyond the diffusion tensor. In: Johannesson-Berg H, Behrens TEJ, eds. Diffusion MRI: from Quantitative Measurement to In Vivo Neuroanatomy. Cambridge MA USA: Academic Press; 2009:56-74.

28. Fontana F. Traité sur le Vénin de la Vipere, sur les Poisons Americains. chez Nyon l'Ainé: Florence; 1781.

29. Dyrby TB, Burke M, Alexander DC, Ptito M. Undulating and crossing axons in the corpus callosum may explain the overestimation of axon diameters with ActiveAx. Paper presented at: ISMRM 23rd Annual Meeting and Exhibition; May 10-16, 2014; Milan, Italy; 2619.

30. Hering H, Sheng M. Dendritic spines: structure, dynamics and regulation. Nat Rev Neurosci. 2001;2:880-888.

31. Schuz A, Braitenberg V. The human cortical white matter: quantitative aspects of cortico-cortical long-range connectivity. In: Schuz A, Miller R, eds. Cortical Areas: Unity and Diversity. London: Taylor-Francis; 2002:377-385.

32. Aboitiz F, Scheibel AB, Fisher RS, Zaidel E. Fiber composition of the human corpus callosum. Brain Res. 1992;598:143-153.

33. Lamantia AS, Rakic P. Cytological and quantitative characteristics of four cerebral commissures in the rhesus monkey. J Comp Neurol. 1990;291:520-537.

34. Ong HH, Wright AC, Wehrli SL, et al. Indirect measurement of regional axon diameter in excised mouse spinal cord with $q$-space imaging: simulation and experimental studies. Neuroimage. 2008;40:1619-1632.

35. Waxman SG, Bennett MV. Relative conduction velocities of small myelinated and non-myelinated fibres in the central nervous system. Nat New Biol. 1972;238:217-219.

36. Perge JA, Koch K, Miller R, Sterling P, Balasubramanian V. How the optic nerve allocates space, energy capacity, and information. J Neurosci. 2009;29:7917-7928.

37. Innocenti GM, Vercelli A, Caminiti R. The diameter of cortical axons depends both on the area of origin and target. Cereb Cortex. 2014;24:2178-2188.

38. Innocenti GM, Carlen M, Dyrby TB. The diameters of cortical axons and their relevance to neural computing. In: Rockland KS, ed. Axons and Brain Architecture. Cambridge MA USA: Academic Press; 2015:319-337.

39. Caminiti R, Ghaziri H, Galuske R, Hof PR, Innocenti GM. Evolution amplified processing with temporally dispersed slow neuronal connectivity in primates. Proc Natl Acad Sci U S A. 2009;106:19551-19556. 
40. Sepehrband F, Alexander DC, Clark KA, Kurniawan ND, Yang Z, Reutens DC. Parametric probability distribution functions for axon diameters of corpus callosum. Front Neuroanat. 2016;10:59

41. Riise J, Pakkenberg B. Stereological estimation of the total number of myelinated callosal fibers in human subjects. J Anat. 2011;218:277-284.

42. Schmahmann JD, Pandya DN. Fiber Pathways of the Brain. New York: Oxford University Press; 2006.

43. Innocenti GM, Dyrby TB, Andersen KW, Rouiller EM, Caminiti R. The crossed projection to the striatum in two species of monkey and in humans: behavioral and evolutionary significance. Cereb Cortex. 2016;27(6):3217-3230.

44. Innocenti GM, Caminiti R. Axon diameter relates to synaptic bouton size: structural properties define computationally different types of cortical connections in primates. Brain Struct Funct. 2016;222(3):1169-1177.

45. Tomasi S, Caminiti R, Innocenti GM. Areal differences in diameter and length of corticofugal projections. Cereb Cortex. 2012;22:1463-1472.

46. Rushton WA. A theory of the effects of fibre size in medullated nerve. J Physiol. 1951;115:101-122.

47. Salzer JL. Clustering sodium channels at the node of Ranvier: close encounters of the axon-glia kind. Neuron. 1997;18:843-846.

48. Smith RS, Koles ZJ. Myelinated nerve fibers: computed effect of myelin thickness on conduction velocity. Am J Physiol. 1970;219:1256-1258.

49. Fadic R, Vergara J, Alvarez J. Microtubules and caliber of central and peripheral processes of sensory axons. J Comp Neurol. 1985;236:258-264.

50. Pelvig DP, Pakkenberg H, Stark AK, Pakkenberg B. Neocortical glial cell numbers in human brains. Neurobiol Aging. 2008;29:1754-1762.

51. Salvesen L, Winge K, Brudek T, Agander TK, Lokkegaard A, Pakkenberg B. Neocortical neuronal loss in patients with multiple system atrophy: a stereological study. Cereb Cortex. 2017;27:400-410.

52. Manley GT, Binder DK, Papadopoulos MC, Verkman AS. New insights into water transport and edema in the central nervous system from phenotype analysis of aquaporin-4 null mice. Neuroscience. 2004;129:983-991.

53. Baumann N, Pham-Dinh D. Biology of oligodendrocyte and myelin in the mammalian central nervous system. Physiol Rev. 2001;81:871-927.

54. Bakiri Y, Karadottir R, Cossell L, Attwell D. Morphological and electrical properties of oligodendrocytes in the white matter of the corpus callosum and cerebellum. J Physiol. 2011;589:559-573.

55. Oberheim NA, Takano T, Han X, et al. Uniquely hominid features of adult human astrocytes. J Neurosci. 2009;29:3276-3287.

56. Ding F, O'Donnell J, Xu Q, Kang N, Goldman N, Nedergaard M. Changes in the composition of brain interstitial ions control the sleep-wake cycle. Science. 2016;352:550-555.

57. Khakh BS, Sofroniew MV. Diversity of astrocyte functions and phenotypes in neural circuits. Nat Neurosci. 2015;18:942-952.

58. Gehrmann J, Matsumoto Y, Kreutzberg GW. Microglia: intrinsic immuneffector cell of the brain. Brain Res Rev. 1995;20:269-287.

59. Kettenmann H, Verkhratsky A. Neuroglia-living nerve glue. Fortschr Neurol Psychiatr. 2011;79:588-597.

60. Sykova E, Nicholson C. Diffusion in brain extracellular space. Physiol Rev. 2008;88:1277-1340.

61. Houzel JC, Milleret C, Innocenti G. Morphology of callosal axons interconnecting areas 17 and 18 of the cat. Eur J Neurosci. 1994;6:898-917.

62. Lundell H, Nielsen JB, Ptito M, Dyrby TB. Distribution of collateral fibers in the monkey cervical spinal cord detected with diffusion-weighted magnetic resonance imaging. Neuroimage. 2011;56:923-929.

63. Nilsson M, Latt J, Stahlberg F, van Westen D, Hagslatt H. The importance of axonal undulation in diffusion MR measurements: a Monte Carlo simulation study. NMR Biomed. 2012;25:795-805.

64. Weber N, Keller AL, Reichold J, Logothetis NK. The microvascular system of the striate and extrastriate visual cortex of the macaque. Cereb Cortex. 2008;18:2318-2330.

65. Le Bihan D, Breton E, Lallemand D, Aubin ML, Vignaud J, Laval-Jeantet M. Separation of diffusion and perfusion in intravoxel incoherent motion MR imaging. Radiology. 1988;168(2):

66. Stejskal EO, Tanner JE. Spin diffusion measurements: spin echoes in the presence of a time-dependent field gradient. J Chem Phys. 1965;42:288

67. Shemesh N, Jespersen SN, Alexander DC, et al. Conventions and nomenclature for double diffusion encoding NMR and MRI. Magn Reson Med. 2016;75:82-87.

68. Assaf Y, Mayk A, Cohen Y. Displacement imaging of spinal cord using q-space diffusion-weighted MRI. Magn Reson Med. 2000;44:7137-7122.

69. Stanisz GJ, Szafer A, Wright GA, Henkelman RM. An analytical model of restricted diffusion in bovine optic nerve. Magn Reson Med. 1997;37:103-111.

70. Aslund I, Nowacka A, Nilsson M, Topgaard D. Filter-exchange PGSE NMR determination of cell membrane permeability. J Magn Reson. 2009;200: 291-295.

71. Reese TG, Heid O, Weisskoff RM, Wedeen VJ. Reduction of eddy-current-induced distortion in diffusion MRI using a twice-refocused spin echo. Magn Reson Med. 2003;49:177-182.

72. Finsterbusch J. Eddy-current compensated diffusion weighting with a single refocusing RF pulse. Magn Reson Med. 2009;61:748-754.

73. Tanner JE. Use of the stimulated echo in NMR diffusion studies. J Chem Phys. 1970;52:2523

74. Merboldt KD, Hanicke W, Frahm J. Diffusion imaging using stimulated echoes. Magn Reson Med. 1991;19:233-239.

75. Schick F. Signal losses in diffusion preparation: comparison between spin-echo, stimulated echo and SEASON. Magn Reson Mater Phys Biol Med. 1998;6:53-61.

76. Lundell H, Alexander DC, Dyrby TB. High angular resolution diffusion imaging with stimulated echoes: compensation and correction in experiment design and analysis. NMR Biomed. 2014;27:918-925.

77. Laun FB, Kuder TA, Semmler W, Stieltjes B. Determination of the defining boundary in nuclear magnetic resonance diffusion experiments. Phys Rev Lett. 2011;107: 048102

78. Kaden E, Knosche TR, Anwander A. Parametric spherical deconvolution: inferring anatomical connectivity using diffusion MR imaging. Neuroimage. 2007;37:474-488.

79. Jespersen SN, Kroenke CD, Ostergaard L, Ackerman JJ, Yablonskiy DA. Modeling dendrite density from magnetic resonance diffusion measurements. Neuroimage. 2007;34:1473-1486. 
80. Callaghan PT. Principles of Nuclear Magnetic Resonance. Oxford: Oxford University Press; 1991.

81. Packer KJ, Rees C, Pulsed NMR. studies of restricted diffusion. I. Droplet size distributions in emulsions. J Colloid Interface Sci. 1972;40:206-218.

82. Andrasko J. Water diffusion permeability of human erythrocytes studied by a pulsed gradient NMR technique. Biochim Biophys Acta. 1976;428: 304-311.

83. Kärger J, Zur Bestimmung d. Diffusion in einem Zweibereichsystem mit Hilfe von gepulsten Feldgradienten. Ann Phys. 1969;479:1-4.

84. Nilsson M, Alerstam E, Wirestam R, Stahlberg F, Brockstedt S, Latt J. Evaluating the accuracy and precision of a two-compartment Karger model using Monte Carlo simulations. J Magn Reson. 2010;206:59-67.

85. Ahlgren A, Knutsson L, Wirestam R, et al. Quantification of microcirculatory parameters by joint analysis of flow-compensated and non-flow-compensated intravoxel incoherent motion (IVIM) data. NMR Biomed. 2016;29:640-649.

86. Cory DG, Garroway AN, Miller JB. Applications of spin transport as a probe of local geometry. Abstracts Papers Am Chem Soc. 1990;199:105-POLY.

87. Callaghan PT, Komlosh ME. Locally anisotropic motion in a macroscopically isotropic system: displacement correlations measured using double pulsed gradient spin-echo NMR. Magn Reson Chem. 2002;40:S15-S19.

88. Koch MA, Finsterbusch J. Compartment size estimation with double wave vector diffusion-weighted imaging. Magn Reson Med. 2008;60:90-101.

89. Finsterbusch J. The parallel-antiparallel signal difference in double-wave-vector diffusion-weighted MR at short mixing times: a phase evolution perspective. J Magn Reson. 2011;208:114-121.

90. Jespersen SN, Lundell H, Sonderby CK, Dyrby TB. Orientationally invariant metrics of apparent compartment eccentricity from double pulsed field gradient diffusion experiments. NMR Biomed. 2013;26:1647-1662.

91. Ozarslan E, Basser PJ. Microscopic anisotropy revealed by NMR double pulsed field gradient experiments with arbitrary timing parameters. $J$ Chem Phys. 2008;128: 154511

92. Shemesh N, Ozarslan E, Adiri T, Basser PJ, Cohen Y. Noninvasive bipolar double-pulsed-field-gradient NMR reveals signatures for pore size and shape in polydisperse, randomly oriented, inhomogeneous porous media. J Chem Phys. 2010;133: 044705

93. Furo I, Dvinskikh SV. NMR methods applied to anisotropic diffusion. Magn Reson Chem. 2002;40:S3-S14.

94. Lasic S, Nilsson M, Latt J, Stahlberg F, Topgaard D. Apparent exchange rate mapping with diffusion MRI. Magn Reson Med. 2011;66:356-365.

95. Sønderby CK, Lundell H, Dyrby TB. Assessing exchange between multiple compartments using multi-directional double wave diffusion sequences. Proc Int Soc Magn Reson Med. 2012;352:

96. Fujita N, Harada K, Sakurai K, Akai Y, Kozuka T. Separation of diffusion and slow flow effects by use of flow rephasing and dephasing. Magn Reson Med. 1992;24:109-122.

97. Wetscherek A, Stieltjes B, Laun FB. Flow-compensated intravoxel incoherent motion diffusion imaging. Magn Reson Med. 2015;74:410-419.

98. Shemesh N, Ozarslan E, Basser PJ, Cohen Y. Accurate noninvasive measurement of cell size and compartment shape anisotropy in yeast cells using double-pulsed field gradient MR. NMR Biomed. 2012;25:236-246.

99. Ozarslan E, Basser PJ. MR diffusion-'diffraction' phenomenon in multi-pulse-field-gradient experiments. J Magn Reson. 2007;188:285-294.

100. Kuder TA, Laun FB. NMR-based diffusion pore imaging by double wave vector measurements. Magn Reson Med. 2012;70:836-841.

101. Shemesh N, Ozarslan E, Basser PJ, Cohen Y. Detecting diffusion-diffraction patterns in size distribution phantoms using double-pulsed field gradient NMR: theory and experiments. J Chem Phys. 2010;132: 034703

102. Shemesh N, Westin C-F, Cohen Y. Magnetic resonance imaging by synergistic diffusion-diffraction patterns. Phys Rev Lett. 2012;108: 058103

103. Callaghan PT, Stepisnik J. Frequency-domain analysis of spin motion using modulated-gradient NMR. J Magn Reson. 1995;117:118-122.

104. Schachter M, Does MD, Anderson AW, Gore JC. Measurements of restricted diffusion using an oscillating gradient spin-echo sequence. J Magn Reson. 2000;147:232-237.

105. Topgaard D, Malmborg C, Soderman O. Restricted self-diffusion of water in a highly concentrated w/o emulsion studied using modulated gradient spinecho NMR. J Magn Reson. 2002;156:195-201.

106. lanus A, Siow B, Drobnjak I, Zhang H, Alexander DC. Gaussian phase distribution approximations for oscillating gradient spin echo diffusion MRI. J Magn Reson. 2013;227:25-34.

107. Shemesh N, Alvarez GA, Frydman L. Size distribution imaging by non-uniform oscillating-gradient spin echo (NOGSE) MRI. PLoS ONE. 2015;10: e0133201

108. Gore JC, Xu J, Colvin DC, Yankeelov TE, Parsons EC, Does MD. Characterization of tissue structure at varying length scales using temporal diffusion spectroscopy. NMR Biomed. 2010;23:745-756.

109. Xu J, Li H, Harkins KD, et al. Mapping mean axon diameter and axonal volume fraction by MRI using temporal diffusion spectroscopy. Neuroimage. 2014;103C:10-19.

110. Drobnjak I, Zhang H, lanus A, Kaden E, Alexander DC. PGSE, OGSE, and sensitivity to axon diameter in diffusion MRI: insight from a simulation study. Magn Reson Med. 2016;75:688-700.

111. Nilsson M, Lasic S, Drobnjak I, Topgaard D, Westin CF. Resolution limit of cylinder diameter estimation by diffusion MRI: the impact of gradient waveform and orientation dispersion. NMR Biomed. 2017;30:e3711. https://doi.org/10.1002/nbm.3711

112. Drobnjak I, Alexander DC. Optimising time-varying gradient orientation for microstructure sensitivity in diffusion-weighted MR. J Magn Reson. 2011;212:344-354.

113. Drobnjak I, Siow B, Alexander DC. Optimizing gradient waveforms for microstructure sensitivity in diffusion-weighted MR. J Magn Reson. 2010;206:41-51.

114. lanus A, Shemesh N, Alexander DC, Drobnjak I. Double oscillating diffusion encoding and sensitivity to microscopic anisotropy. Magn Reson Med. 2016;78(2):550-564.

115. Mitra PP. Multiple wave-vector extensions of the NMR pulsed-field-gradient spin-echo diffusion measurement. Phys Rev B. 1995;51:15074-15078.

116. de Almeida Martins JP, Topgaard D. Two-dimensional correlation of isotropic and directional diffusion using NMR. Phys Rev Lett. 2016;116: 087601 
117. Lundell H, Sonderby CK, Dyrby TB. Diffusion weighted imaging with circularly polarized oscillating gradients. Magn Reson Med. 2015;73:1171-1176.

118. Eriksson S, Lasic S, Topgaard D. Isotropic diffusion weighting in PGSE NMR by magic-angle spinning of the q-vector. J Magn Reson. 2013;226:13-18.

119. Szczepankiewicz F, Lasic S, van Westen D, et al. Quantification of microscopic diffusion anisotropy disentangles effects of orientation dispersion from microstructure: applications in healthy volunteers and in brain tumors. Neuroimage. 2014;104:241-252.

120. Sjolund J, Szczepankiewicz F, Nilsson M, Topgaard D, Westin CF, Knutsson H. Constrained optimization of gradient waveforms for generalized diffusion encoding. J Magn Reson. 2015;261:157-168.

121. Westin CF, Knutsson H, Pasternak O, et al. Q-space trajectory imaging for multidimensional diffusion MRI of the human brain. Neuroimage. 2016;135:345-362.

122. Schmidt-Rohr K, Spiess HW. Multidimensional Solid-State NMR and Polymers. London: Academic Press; 1994.

123. Basser PJ, Mattiello J, Lebihan D. MR diffusion tensor spectroscopy and imaging. Biophys J. 1994;66:259-267.

124. Stikov N, Perry LM, Mezer A, et al. Bound pool fractions complement diffusion measures to describe white matter micro and macrostructure. Neuroimage. 2011;54:1112-1121.

125. Jensen JH, Helpern JA, Ramani A, Lu H, Kaczynski K. Diffusional kurtosis imaging: the quantification of non-gaussian water diffusion by means of magnetic resonance imaging. Magn Reson Med. 2005;53:1432-1440.

126. King MD, Houseman J, Roussel SA, van Bruggen N, Williams SR, Gadian DG. q-Space imaging of the brain. Magn Reson Med. 1994;32:707-713.

127. Wedeen VJ, Hagmann P, Tseng WY, Reese TG, Weisskoff RM. Mapping complex tissue architecture with diffusion spectrum magnetic resonance imaging. Magn Reson Med. 2005;54:1377-1386.

128. Ozarslan E, Koay CG, Shepherd TM, et al. Mean apparent propagator (MAP) MRI: a novel diffusion imaging method for mapping tissue microstructure. Neuroimage. 2013;78:16-32.

129. Finsterbusch J, Koch MA. A tensor approach to double wave vector diffusion-weighting experiments on restricted diffusion. J Magn Reson. 2008;195:23-32.

130. Hui ES, Jensen JH. Double-pulsed diffusional kurtosis imaging for the in vivo assessment of human brain microstructure. Neuroimage. 2015;120:371-381.

131. Jensen JH, Hui ES, Helpern JA. Double-pulsed diffusional kurtosis imaging. NMR Biomed. 2014;27(4):363-370.

132. Niendorf T, Dijkhuizen RM, Norris DG, van Lookeren Campagne M, Nicolay K. Biexponential diffusion attenuation in various states of brain tissue: implications for diffusion-weighted imaging. Magn Reson Med. 1996;36:847-857.

133. Whitaker S. Diffusion and dispersion in porous media. Am Inst Chem Eng J. 1967;13:420-427.

134. Gray WG. A derivation of the equations for mult-phase transport. Chem Eng Sci. 1975;30:229-233.

135. Lehner FK. On the validity of Fick's law for transient diffusion through a porous medium. Chem Eng Sci. 1979;34:821-825.

136. Nicholson C, Phillips JM. Ion diffusion modified by tortuosity and volume fraction in the extracellular microenvironment of the rat cerebellum. $J$ Physiol. 1981;321:225-257.

137. Szafer A, Zhong J, Gore JC. Theoretical model for water diffusion in tissues. Magn Reson Med. 1995;33:697-712.

138. Kärger J, Pfeifer H, Wilfried H. Principles and application of self-diffusion measurements by nuclear magnetic resonance. Adv Magn Reson. 1988;12:1-89.

139. Behrens TE, Woolrich MW, Jenkinson M, et al. Characterization and propagation of uncertainty in diffusion-weighted MR imaging. Magn Reson Med. 2003;50:1077-1088.

140. Assaf Y, Basser PJ. Composite hindered and restricted model of diffusion (CHARMED) MR imaging of the human brain. Neuroimage. 2005;27:48-58.

141. Assaf Y, Freidlin RZ, Rohde GK, Basser PJ. New modeling and experimental framework to characterize hindered and restricted water diffusion in brain white matter. Magn Reson Med. 2004;52:965-978.

142. Fieremans E, Jensen JH, Helpern JA. White matter characterization with diffusional kurtosis imaging. Neuroimage. 2011;58:177-188.

143. Hui ES, Glenn GR, Helpern JA, Jensen JH. Kurtosis analysis of neural diffusion organization. Neuroimage. 2015;106:391-403.

144. Pasternak O, Sochen N, Gur Y, Intrator N, Assaf Y. Free water elimination and mapping from diffusion MRI. Magn Reson Med. 2009;62:717-730.

145. Alexander DC. A general framework for experiment design in diffusion MRI and its application in measuring direct tissue-microstructure features. Magn Reson Med. 2008;60:439-448.

146. Panagiotaki E, Schneider T, Siow B, Hall MG, Lythgoe MF, Alexander DC. Compartment models of the diffusion MR signal in brain white matter: a taxonomy and comparison. Neuroimage. 2012;59:2241-2254.

147. Ferizi U, Schneider T, Panagiotaki E, et al. A ranking of diffusion MRI compartment models with in vivo human brain data. Magn Reson Med. 2014;72:1785-1792.

148. Anderson AW. Measurement of fiber orientation distributions using high angular resolution diffusion imaging. Magn Reson Med. 2005;54:1194-1206.

149. Behrens TE, Berg HJ, Jbabdi S, Rushworth MF, Woolrich MW. Probabilistic diffusion tractography with multiple fibre orientations: what can we gain? Neuroimage. 2007;34:144-155.

150. Sotiropoulos SN, Behrens TEJ, Jbabdi S. Ball and rackets: inferring fiber fanning from diffusion-weighted MRI. Neuroimage. 2012;60:1412-1425.

151. Zhang H, Hubbard PL, Parker GJ, Alexander DC. Axon diameter mapping in the presence of orientation dispersion with diffusion MRI. Neuroimage. 2011;56:1301-1315.

152. Zhang H, Dyrby TB, Alexander DC. Axon diameter mapping in crossing fibers with diffusion MRI. Med Image Comput Comput Assist Interv. 2011;14: 82-89.

153. Barazany D, Jones D, Assaf Y. AxCaliber 3D. Proc Int Soc Magn Reson Med. 2011;19:76

154. Tariq M, Schneider T, Alexander DC, Gandini Wheeler-Kingshott CA, Zhang H. Bingham-NODDI: mapping anisotropic orientation dispersion of neurites using diffusion MRI. Neuroimage. 2016;133:207-223.

155. Farooq H, Xu J, Nam JW, et al. Microstructure imaging of crossing (MIX) white matter fibers from diffusion MRI. Sci Rep. 2016;6: 38927 
156. Jelescu IO, Veraart J, Fieremans E, Novikov DS. Degeneracy in model parameter estimation for multi-compartmental diffusion in neuronal tissue. NMR Biomed. 2016;29:33-47.

157. Wang Y, Wang Q, Haldar JP, et al. Quantification of increased cellularity during inflammatory demyelination. Brain. 2011;134:3590-3601.

158. Jbabdi S, Sotiropoulos SN, Savio AM, Grana M, Behrens TE. Model-based analysis of multishell diffusion MR data for tractography: how to get over fitting problems. Magn Reson Med. 2012;68:1846-1855.

159. White NS, Leergaard TB, D'Arceuil H, Bjaalie JG, Dale AM. Probing tissue microstructure with restriction spectrum imaging: histological and theoretical validation. Hum Brain Mapp. 2013;34:327-346.

160. Scherrer B, Schwartzman A, Taquet M, Sahin M, Prabhu SP, Warfield SK. Characterizing brain tissue by assessment of the distribution of anisotropic microstructural environments in diffusion-compartment imaging (DIAMOND). Magn Reson Med. 2016;76:963-977.

161. Jian B, Vemuri BC, Ozarslan E, Carney PR, Mareci TH. A novel tensor distribution model for the diffusion-weighted MR signal. Neuroimage. 2007;37:164-176.

162. Ramirez-Manzanares A, Rivera M, Vemuri BC, Carney P, Mareci T. Diffusion basis functions decomposition for estimating white matter intravoxel fiber geometry. IEEE Trans Med Imaging. 2007;26:1091-1102.

163. Leow AD, Zhu S, Zhan L, et al. The tensor distribution function. Magn Reson Med. 2009;61:205-214.

164. Assaf Y, Cohen Y. Assignment of the water slow-diffusing component in the central nervous system using q-space diffusion MRS: implications for fiber tract imaging. Magn Reson Med. 2000;43:191-199.

165. Palombo M, Ligneul C, Najac C, et al. New paradigm to assess brain cell morphology by diffusion-weighted MR spectroscopy in vivo. Proc Natl Acad Sci U S A. 2016;113:6671-6676.

166. Nedjati-Gilani GL, Schneider T, Hall MG, Wheeler-Kingshott CA, Alexander DC. Machine learning based compartment models with permeability for white matter microstructure imaging. Med Image Comput Comput Assist Interv. 2014;17:257-264.

167. Silva MD, Helmer KG, Lee JH, Han SS, Springer CS, Sotak CH. Deconvolution of compartmental water diffusion coefficients in yeast-cell suspensions using combined $T_{1}$ and diffusion measurements. J Magn Reson. 2002;156:52-63.

168. Shemesh N, Adiri T, Cohen Y. Probing microscopic architecture of opaque heterogeneous systems using double-pulsed-field-gradient NMR. J Am Chem Soc. 2011;133:6028-6035.

169. Burcaw LM, Fieremans E, Novikov DS. Mesoscopic structure of neuronal tracts from time-dependent diffusion. Neuroimage. 2015;114:18-37.

170. De Santis S, Jones DK, Roebroeck A. Including diffusion time dependence in the extra-axonal space improves in vivo estimates of axonal diameter and density in human white matter. Neuroimage. 2016;130:91-103.

171. Kaden E, Kruggel F, Alexander DC. Quantitative mapping of the per-axon diffusion coefficients in brain white matter. Magn Reson Med. 2016;75: 1752-1763.

172. Hutchinson EB, Avram AV, Irfanoglu MO, et al. Analysis of the effects of noise, DWI sampling, and value of assumed parameters in diffusion MRI models. Magn Reson Med. 2017;78(5):1767-1780.

173. Novikov DS, Veraart J, Jelescu IO, Fieremans E. Rotationally invariant mapping of microstructural and orientational neuronal tissue parameters in human brain. Poster presented at: ISMRM 25th Annual Meeting and Exhibition; April 22-27, 2017; Honolulu, HI.

174. Vestergaard-Poulsen P, Wegener G, Hansen B, et al. Diffusion-weighted MRI and quantitative biophysical modeling of hippocampal neurite loss in chronic stress. PLoS ONE. 2011;6: e20653

175. Wang S, Chopp M, Nazem-Zadeh MR, et al. Comparison of neurite density measured by MRI and histology after TBI. PLoS ONE. $2013 ; 8:$ e63511.

176. Tavor I, Hofstetter S, Assaf Y. Micro-structural assessment of short term plasticity dynamics. Neuroimage. 2013;81:1-7.

177. Kunz N, Zhang H, Vasung L, et al. Assessing white matter microstructure of the newborn with multi-shell diffusion MRI and biophysical compartment models. Neuroimage. 2014;96:288-299.

178. Winston GP, Micallef C, Symms MR, Alexander DC, Duncan JS, Zhang H. Advanced diffusion imaging sequences could aid assessing patients with focal cortical dysplasia and epilepsy. Epilepsy Res. 2014;108:336-339.

179. Eaton-Rosen Z, Melbourne A, Orasanu E, et al. Longitudinal measurement of the developing grey matter in preterm subjects using multi-modal MRI. Neuroimage. 2015;111:580-589.

180. Nazeri A, Chakravarty MM, Rotenberg DJ, et al. Functional consequences of neurite orientation dispersion and density in humans across the adult lifespan. J Neurosci. 2015;35:1753-1762.

181. Chang YS, Owen JP, Pojman NJ, et al. White matter changes of neurite density and fiber orientation dispersion during human brain maturation. PLoS ONE. 2015;10: e0123656

182. Dean DC III, O'Muircheartaigh J, Dirks H, et al. Mapping an index of the myelin g-ratio in infants using magnetic resonance imaging. Neuroimage. 2016;132:225-237.

183. Timmers I, Zhang H, Bastiani M, Jansma BM, Roebroeck A, Rubio-Gozalbo ME. White matter microstructure pathology in classic galactosemia revealed by neurite orientation dispersion and density imaging. J Inherit Metab Dis. 2015;38:295-304.

184. Owen JP, Chang YS, Pojman NJ, et al. Aberrant white matter microstructure in children with 16p11.2 deletions. J Neurosci. 2014;34:6214-6223.

185. Kamagata K, Hatano T, Okuzumi A, et al. Neurite orientation dispersion and density imaging in the substantia nigra in idiopathic Parkinson disease. Eur Radiol. 2016;26:2567-2577.

186. Colgan N, Siow B, O'Callaghan JM, et al. Application of neurite orientation dispersion and density imaging (NODDI) to a tau pathology model of Alzheimer's disease. Neuroimage. 2016;125:739-744.

187. Lemkaddem A, Daducci A, Kunz N, et al. Connectivity and tissue microstructural alterations in right and left temporal lobe epilepsy revealed by diffusion spectrum imaging. Neurolmage Clin. 2014;5:349-358.

188. Billiet T, Madler B, D'Arco F, et al. Characterizing the microstructural basis of 'unidentified bright objects' in neurofibromatosis type 1: a combined in vivo multicomponent T2 relaxation and multi-shell diffusion MRI analysis. Neurolmage Clin. 2014;4:649-658. 
189. Jelescu IO, Veraart J, Adisetiyo V, Milla SS, Novikov DS, Fieremans E. One diffusion acquisition and different white matter models: how does microstructure change in human early development based on WMTI and NODDI? Neuroimage. 2015;107:242-256.

190. Gao J, Li X, Li Y, et al. Differentiating T2 hyperintensity in neonatal white matter by two-compartment model of diffusional kurtosis imaging. Sci Rep. 2016;6: 24473

191. Fieremans E, Benitez A, Jensen JH, et al. Novel white matter tract integrity metrics sensitive to Alzheimer disease progression. Am J Neuroradiol. 2013;34:2105-2112.

192. Benitez A, Fieremans $\mathrm{E}$, Jensen $\mathrm{JH}$, et al. White matter tract integrity metrics reflect the vulnerability of late-myelinating tracts in Alzheimer's disease. Neurolmage Clin. 2014;4:64-71.

193. Guglielmetti C, Veraart J, Roelant E, et al. Diffusion kurtosis imaging probes cortical alterations and white matter pathology following cuprizone induced demyelination and spontaneous remyelination. Neuroimage. 2016;125:363-377.

194. Jelescu IO, Zurek M, Winters KV, et al. In vivo quantification of demyelination and recovery using compartment-specific diffusion MRI metrics validated by electron microscopy. Neuroimage. 2016;132:104-114.

195. Kelm ND, West KL, Carson RP, Gochberg DF, Ess KC, Does MD. Evaluation of diffusion kurtosis imaging in ex vivo hypomyelinated mouse brains. Neuroimage. 2016;124:612-626.

196. Horowitz A, Barazany D, Tavor I, Bernstein M, Yovel G, Assaf Y. In vivo correlation between axon diameter and conduction velocity in the human brain. Brain Struct Funct. 2015;220:1777-1788.

197. Innocenti GM, Caminiti R, Aboitiz F. Comments on the paper by Horowitz et al. Brain Struct Funct. 2015;220:1789-1790.

198. Huang SY, Tobyne SM, Nummenmaa A, et al. Characterization of axonal disease in patients with multiple sclerosis using high-gradient-diffusion MR imaging. Radiology. 2016;280:244-251.

199. Burnham KP, Anderson DR. Model Selection and Multimodal Inference. New York: Springer; 2002.

200. Mackay DJC. Information Theory, Inference, and Learning Algorithms. Cambridge UK: Cambridge University Press; 2003.

201. Arlot SA. survey of cross-validation procedures for model selection. Stat Surv. 2010;4:40-79.

202. Ferizi U, Schneider T, Witzel T, et al. White matter compartment models for in vivo diffusion MRI at 300 mT/m. Neuroimage. 2015;118:468-483.

203. Ferizi U, Scherrer B, Schneider T, et al. Diffusion MRI microstructure models with in vivo human brain Connectom data: results from a multi-group comparison. NMR Biomed. 2017;30:e3734. https://doi.org/10.1002/nbm.3734

204. Ghosh A, Alexander DC, Zhang H. To be dispersed or not to be dispersed: a study using HCP data. Poster presented at: ISMRM $24^{\text {th }}$ Annual Meeting and Exhibition; May 7-13, 2016; Singapore.

205. Rokem A, Yeatman JD, Pestilli F, et al. Evaluating the accuracy of diffusion MRI models in white matter. PLoS ONE. 2015;10:e0123272.

206. Nilsson M, Latt J, Nordh E, Wirestam R, Stahlberg F, Brockstedt S. On the effects of a varied diffusion time in vivo: is the diffusion in white matter restricted? Magn Reson Imaging. 2009;27:176-187.

207. Lindgren BW. Statistical Theory. New York: Chapman and Hall; 1993.

208. Pukelsheim F. Optimal Design of Experiments. New York: Wiley; 1993.

209. Brihuega-Moreno O, Heese FP, Hall LD. Optimization of diffusion measurements using Cramer-Rao lower bound theory and its application to articular cartilage. Magn Reson Med. 2003;50:1069-1076.

210. Cercignani M, Alexander DC. Optimal acquisition schemes for in vivo quantitative magnetization transfer MRI. Magn Reson Med. 2006;56:803-810.

211. Xie J, Gallichan D, Gunn RN, Jezzard P. Optimal design of pulsed arterial spin labeling MRI experiments. Magn Reson Med. 2008;59:826-834.

212. Lampinen B, Szczepankiewicz F, van Westen D, et al. Optimal experimental design for filter exchange imaging: apparent exchange rate measurements in the healthy brain and in intracranial tumors. Magn Reson Med. 2016;77(3):1104-1114. https://doi.org/10.1002/mrm.26195

213. Nilsson M, Lätt J, van Westen D, et al. Noninvasive mapping of water diffusional exchange in the human brain using filter-exchange imaging. Magn Reson Med. 2013;69:1572-1580.

214. Siow B, Drobnjak I, Chatterjee A, Lythgoe MF, Alexander DC. Estimation of pore size in a microstructure phantom using the optimised gradient waveform diffusion weighted NMR sequence. J Magn Reson. 2012;214:51-60.

215. Callaghan PT, Jolley KW, Lelievre J. Diffusion of water in the endosperm tissue of wheat grains as studied by pulsed field gradient nuclear magnetic resonance. Biophys J. 1979;28:133-141.

216. Lindblom G, Wennerstrom H, Arvidson G. Translational diffusion in model membranes studied by nuclear magnetic resonance. Biophysical Chemistry. 1977;6(2):167-171.

217. Kroenke CD, Ackerman JJ, Yablonskiy DA. On the nature of the NAA diffusion attenuated MR signal in the central nervous system. Magn Reson Med. 2004;52:1052-1059.

218. Lasic S, Szczepankiewicz F, Eriksson S, Nilsson M, Topgaard D. Microanisotropy imaging: quantification of microscopic diffusion anisotropy and orientational order parameter by diffusion MRI with magic-angle spinning of the q-vector. Front Phys. 2014;2:11

219. Reisert M, Kellner E, Dhital B, Hennig J, Kiselev VG. Disentangling micro from mesostructure by diffusion MRI: a Bayesian approach. Neuroimage. 2017;147:964-975.

220. Nagy Z, Alexander DC, Thomas DL, Weiskopf N, Sereno MI. Using high angular resolution diffusion imaging data to discriminate cortical regions. PLoS ONE. 2013;8: e63842

221. Daducci A, Canales-Rodriguez EJ, Zhang H, Dyrby TB, Alexander DC, Thiran JP. Accelerated microstructure imaging via convex optimization (AMICO) from diffusion MRI data. Neuroimage. 2015;105:32-44.

222. Novikov DS, Jelescu IO, Fieremans E. From diffusion signal moments to neurite diffusivities, volume fraction and orientation distribution: an exact solution. Paper presented at: ISMRM $23^{\text {rd }}$ Annual Meeting and Exhibition; June 2, 2015; Toronto; 469.

223. Sepehrband F, Alexander DC, Kurniawan ND, Reutens DC, Yang Z. Towards higher sensitivity and stability of axon diameter estimation with diffusionweighted MRI. NMR Biomed. 2016;29:293-308. 
224. Morgan GL. Regional Variation Models of White Matter Microstructure [dissertation]. Science, University College London; 2012.

225. Powell MJD. The BOBYQA Algorithm for Bound Constrained Optimization Without Derivatives. Cambridge: Department of Applied Mathematics and Theoretical Physics; 2009.

226. Reisert M, HKiselev VG, Dihtal B, Kellner E, Novikov DS. MesoFT: unifying diffusion modelling and fiber tracking. Med Image Comput Comput Assist Interv. 2014;17:201-208.

227. Sherbondy AJ, Rowe MC, Alexander DC. MicroTrack: an algorithm for concurrent projectome and microstructure estimation. Med Image Comput Comput Assist Interv. 2010;13:183-190.

228. Hall MG, Alexander DC. Convergence and parameter choice for Monte-Carlo simulations of diffusion MRI. IEEE Trans Med Imaging. 2009;28:1354-1364.

229. Hubbard PL, Zhou FL, Eichhorn SJ, Parker GJ. Biomimetic phantom for the validation of diffusion magnetic resonance imaging. Magn Reson Med. 2015;73:299-305.

230. Latt J, Nilsson M, Rydhog A, Wirestam R, Stahlberg F, Brockstedt S. Effects of restricted diffusion in a biological phantom: a q-space diffusion MRI study of asparagus stems at a 3T clinical scanner. Magn Reson Mater Phys Biol Med. 2007;20:213-222.

231. Jones DK, Basser PJ. 'Squashing peanuts and smashing pumpkins': how noise distorts diffusion-weighted MR data. Magn Reson Med. 2004;52:979-993.

232. Xu J, Does MD, Gore JC. Sensitivity of MR diffusion measurements to variations in intracellular structure: effects of nuclear size. Magn Reson Med. 2009;61:828-833.

233. Alexander AL, Hasan KM, Lazar M, Tsuruda JS, Parker DL. Analysis of partial volume effects in diffusion-tensor MRI. Magn Reson Med. 2001;45:770-780.

234. Vos SB, Jones DK, Viergever MA, Leemans A. Partial volume effect as a hidden covariate in DTI analyses. Neuroimage. 2011;55:1566-1576.

235. Balls GT, Frank LR. A simulation environment for diffusion weighted MR experiments in complex media. Magn Reson Med. 2009;62:771-778.

236. Ford JC, Hackney DB. Numerical model for calculation of apparent diffusion coefficients (ADC) in permeable cylinders-comparison with measured ADC in spinal cord white matter. Magn Reson Med. 1997;37:387-394.

237. Panagiotaki E, Hall MG, Zhang H, Siow B, Lythgoe MF, Alexander DC. High-fidelity meshes from tissue samples for diffusion MRI simulations. Med Image Comput Comput Assist Interv. 2010;13:404-411.

238. Avram L, Ozarslan E, Assaf Y, Bar-Shir A, Cohen Y, Basser PJ. Three-dimensional water diffusion in impermeable cylindrical tubes: theory versus experiments. NMR Biomed. 2008;21:888-898.

239. Shemesh N, Ozarslan E, Basser PJ, Cohen Y. Measuring small compartmental dimensions with low- $q$ angular double-PGSE NMR: the effect of experimental parameters on signal decay. J Magn Reson. 2009;198:15-23.

240. Hua L, Gore JC, Xu J. Fast androbust measurement of microstructural dimensions using temporal diffusion spectroscopy. J Magn Reson. 2014;242:4-9.

241. Komlosh ME, Horkay F, Freidlin RZ, Nevo U, Assaf Y, Basser PJ. Detection of microscopic anisotropy in gray matter and in a novel tissue phantom using double pulsed gradient spin echo MR. J Magn Reson. 2007;189:38-45.

242. Greiner A, Wendorff JH. Electrospinning: a fascinating method for the preparation of ultrathin fibers. Angew Chem Int Ed Engl. 2007;46:5670-5703.

243. Topgaard D. Director orientations in lyotropic liquid crystals: diffusion MRI mapping of the Saupe order tensor. Phys Chem Chem Phys. 2016;18: 8545-8553.

244. Boujraf S, Luypaert R, Eisendrath H, Osteaux M. Echo planar magnetic resonance imaging of anisotropic diffusion in asparagus stems. Magn Reson Mater Phys Biol Med. 2001;13:82-90.

245. Hakansson B, Pons R, Soderman O. Diffraction-like effects in a highly concentrated W/O emulsion: a PFG NMR study. Magn Reson Imaging. 1998;16:643-646.

246. Proverbio A, Siow BM, Lythgoe MF, Alexander DC, Gibson AP. Multimodality characterization of microstructure by the combination of diffusion NMR and time-domain diffuse optical data. Phys Med Biol. 2014;59:2639-2658.

247. Suh KJ, Hong YS, Skirda VD, Volkov VI, Lee CY, Lee CH. Water self-diffusion behavior in yeast cells studied by pulsed field gradient NMR. Biophys Chem. 2003;104:121-130.

248. Malmborg C, Sjobeck M, Brockstedt S, Englund E, Soderman O, Topgaard D. Mapping the intracellular fraction of water by varying the gradient pulse length in q-space diffusion MRI. J Magn Reson. 2006;180:280-285.

249. Dyrby TB, Baare WFC, Alexander DC, Jelsing J, Garde E, Sogaard LV. An ex vivo imaging pipeline for producing high-quality and high-resolution diffusion-weighted imaging datasets. Hum Brain Mapp. 2011;32:544-563.

250. D'Arceuil HE, Westmoreland S, de Crespigny AJ. An approach to high resolution diffusion tensor imaging in fixed primate brain. Neuroimage. 2007;35:553-565.

251. Richardson S, Siow B, Panagiotaki E, Schneider T, Lythgoe MF, Alexander DC. Viable and fixed white matter: diffusion magnetic resonance comparisons and contrasts at physiological temperature. Magn Reson Med. 2014;72:1151-1161.

252. Richardson S, Siow B, Batchelor AM, Lythgoe MF, Alexander DC. Aviable isolated tissue system: a tool for detailed MR measurements and controlled perturbation in physiologically stable tissue. Magn Reson Med. 2013;69:1603-1610.

253. Shepherd TM, Scheffler B, King MA, Stanisz GJ, Steindler DA, Blackband SJ. MR microscopy of rat hippocampal slice cultures: a novel model for studying cellular processes and chronic perturbations to tissue microstructure. Neuroimage. 2006;30:780-786.

254. Choe AS, Stepniewska I, Colvin DC, Ding Z, Anderson AW. Validation of diffusion tensor MRI in the central nervous system using light microscopy: quantitative comparison of fiber properties. NMR Biomed. 2012;25:900-908.

255. Zetterling M, Roodakker KR, Berntsson SG, et al. Extension of diffuse low-grade gliomas beyond radiological borders as shown by the coregistration of histopathological and magnetic resonance imaging data. J Neurosurg. 2016;125:1155-1166.

256. Szczepankiewicz F, van Westen D, Englund E, et al. The link between diffusion MRI and tumor heterogeneity: mapping cell eccentricity and density by diffusional variance decomposition (DIVIDE). Neuroimage. 2016;142:522-532. https://doi.org/10.1016/j.neuroimage.2016.07.038

257. Setsompop K, Kimmlingen R, Eberlein E, et al. Pushing the limits of in vivo diffusion MRI for the Human Connectome Project. Neuroimage. 2013;80:220-233. 
258. Heidemann RM, Porter DA, Anwander A, et al. Diffusion imaging in humans at 7T using readout-segmented EPI and GRAPPA. Magn Reson Med. 2010;64:9-14.

259. Vu AT, Auerbach E, Lenglet C, et al. High resolution whole brain diffusion imaging at 7T for the Human Connectome Project. Neuroimage. 2015;122:318-331.

260. Sotiropoulos SN, Hernandez-Fernandez M, Vu AT, et al. Fusion in diffusion MRI for improved fibre orientation estimation: an application to the $3 T$ and 7T data of the Human Connectome Project. Neuroimage. 2016;134:396-409.

261. Stikov N, Campbell JS, Stroh T, et al. In vivo histology of the myelin g-ratio with magnetic resonance imaging. Neuroimage. 2015;118:397-405.

262. Melbourne A, Eaton-Rosen Z, De vita E, et al. Multi-modal measurement of myelin-to-axon diameter g-ratio in preterm-born neonates and adult controls. Paper presented at: MICCAI 2014; Boston, MA

263. Kim D, Doyle EK, Wisnowski JL, Kim JH, Haldar JP. Diffusion-relaxation correlation spectroscopic imaging: a multidimensional approach for probing microstructure. Magn Reson Med. 2017;78:2236-2249.

264. Kaden E, Barskaya I, Kelm ND, West KL, Does MD, Alexander DC. Microscopic susceptibility anisotropy mapping. Paper presented at: ISMRM $25^{\text {th }}$ Annual Meeting and Exhibition; April 25-27, 2017; Honolulu, HI.

265. Panagiotaki E, Walker-Samuel S, Siow B, et al. Non-invasive quantification of solid tumour microstructure using VERDICT MRI. Cancer Res. 2014;74:1902-1912.

266. Panagiotaki E, Chan RW, Dikaios N, et al. Microstructural characterization of normal and malignant human prostate tissue with vascular, extracellular, and restricted diffusion for cytometry in tumours magnetic resonance imaging. Invest Radiol. 2015;50:218-227.

267. White NS, McDonald C, Farid N, et al. Diffusion-weighted imaging in cancer: physical foundations and applications of restriction spectrum imaging. Cancer Res. 2014;74:4638-4652.

How to cite this article: Alexander DC, Dyrby TB, Nilsson M, Zhang H. Imaging brain microstructure with diffusion MRI: practicality and applications. NMR in Biomedicine. 2017;e3841. https://doi.org/10.1002/nbm.3841 